\title{
FURTHER PROPERTIES OF OSLER'S GENERALIZED FRACTIONAL INTEGRALS AND DERIVATIVES WITH RESPECT TO ANOTHER FUNCTION
}

\author{
RICARDO ALMEIDA
}

\begin{abstract}
In this paper we discuss fractional integrals and fractional derivatives of a function with respect to another function. We present some fundamental properties for both types of fractional operators, such as Taylor's theorem, Leibniz and semigroup rules. We also provide a numerical tool to deal with these operators, by approximating them with a sum involving integer-order derivatives.
\end{abstract}

1. Introduction. Fractional calculus is an important research field, not only in pure mathematics, but in applied mathematics, physics, biology, engineering, economics, etc., as well. In fact, by considering derivatives and integrals of arbitrary real or complex order, we may model more efficiently certain real phenomena. Applications have been found, e.g., in human body modeling $[\mathbf{9}, \mathbf{1 9}]$, heat conduction $[\mathbf{8}]$, viscoelasticity $[\mathbf{1 2}, \mathbf{3 0}]$, time series analysis $[\mathbf{3}, \mathbf{2 9}]$, circuits [21], material sciences [28], shear waves [7], etc.

The subject is as old as calculus itself, and goes back to Leibniz and L'Hôpital, when the meaning of the derivative of order $1 / 2$ was discussed. Since then, many definitions of fractional integrals and fractional derivatives have appeared, each one with its own advantages $[1,16,31]$. However, fractional operators do not share the same properties and because of this we find a large number of works for similar problems. One way to overcome this issue is to define general forms of fractional integrals and fractional derivatives, by considering

2010 AMS Mathematics subject classification. Primary: 26A33, 26A24, 41A58.

Keywords and phrases. Fractional integral, fractional derivative, Taylor's theorem, semigroup law, expansion formulas.

Work supported by Portuguese funds through the CIDMA — Center for Research and Development in Mathematics and Applications, and the Portuguese Foundation for Science and Technology (FCT-Fundação para a Ciência e a Tecnologia), within project UID/MAT/04106/2019. The author is grateful to an anonymous referee for helpful comments and suggestions.

Received by the editors on September 5, 2018, and in revised form on February 13, 2019. 
the derivative and integral with respect to another function $\psi$. For some choices of $\psi$, we obtain some well known definitions, like the Riemann-Liouville $[10,15,18,20,35]$, the Hadamard $[4,5,13]$ and the Erdélyi-Kober fractional operators $[\mathbf{1 4}, \mathbf{3 2}]$. This approach is not very common yet, and much work remains to be done. We mention the papers $[\mathbf{2 2}, \mathbf{2 3}, \mathbf{2 6}]$, where a similar concept of fractional derivative was studied, and a Leibniz and chain formulas were proven. In [6], a Taylor's formula is extended to the same type of derivative operator, and in [11], summation formulas are obtained by using the generalized chain rule. In $[\mathbf{1 7}, \mathbf{3 4}]$, that concept of fractional derivative is generalized by means of a representation based on the Pochhammer's contour of integration.

Throughout this paper, except otherwise stated, $\alpha$ denotes a positive real number, and $n:=[\alpha]+1$ is an integer number. Also, $f \in L_{1}[a, b]$ is a function and $\psi \in C^{1}[a, b]$ is another function satisfying the condition $\psi^{\prime}(x)>0$, for every $x \in[a, b]$. We start by reviewing the definitions needed in the sequel, which can be found, e.g., in $[\mathbf{1 6}, \mathbf{3 1}]$.

Definition 1. [16] The left and right Riemann-Liouville fractional integrals of $f$ of order $\alpha>0$, with respect to function $\psi$, are given by

$$
I_{a+}^{\alpha, \psi} f(x):=\frac{1}{\Gamma(\alpha)} \int_{a}^{x} \psi^{\prime}(t)(\psi(x)-\psi(t))^{\alpha-1} f(t) d t,
$$

for $x \geq a$, and

$$
I_{b-}^{\alpha, \psi} f(x):=\frac{1}{\Gamma(\alpha)} \int_{x}^{b} \psi^{\prime}(t)(\psi(t)-\psi(x))^{\alpha-1} f(t) d t,
$$

for $x \leq b$, respectively.

For example, for $\beta>0$, we have the two following formulas (see [16, Property 2.18]):

$$
I_{a+}^{\alpha, \psi}(\psi(x)-\psi(a))^{\beta-1}=\frac{\Gamma(\beta)}{\Gamma(\beta+\alpha)}(\psi(x)-\psi(a))^{\beta+\alpha-1}
$$

and

$$
I_{b-}^{\alpha, \psi}(\psi(b)-\psi(x))^{\beta-1}=\frac{\Gamma(\beta)}{\Gamma(\beta+\alpha)}(\psi(b)-\psi(x))^{\beta+\alpha-1} .
$$


The semigroup property is valid for fractional integrals. Given $\beta>0$, and a continuous function $f$, the following relations hold (see [16, Lemma 2.26]):

$$
I_{a+}^{\alpha, \psi} I_{a+}^{\beta, \psi} f(x)=I_{a+}^{\alpha+\beta, \psi} f(x) \quad \text { and } \quad I_{b-}^{\alpha, \psi} I_{b-}^{\beta, \psi} f(x)=I_{b-}^{\alpha+\beta, \psi} f(x) .
$$

For what concerns fractional derivatives, we also have the left and right fractional operators.

Definition 2. [16] The left and right Riemann-Liouville fractional derivatives of $f$ of order $\alpha>0$, with respect to function $\psi$, are given by

$$
\begin{aligned}
D_{a+}^{\alpha, \psi} f(x) & :=\left(\frac{1}{\psi^{\prime}(x)} \frac{d}{d x}\right)^{n} I_{a+}^{n-\alpha, \psi} f(x) \\
& =\frac{1}{\Gamma(n-\alpha)}\left(\frac{1}{\psi^{\prime}(x)} \frac{d}{d x}\right)^{n} \int_{a}^{x} \psi^{\prime}(t)(\psi(x)-\psi(t))^{n-\alpha-1} f(t) d t,
\end{aligned}
$$

for $x \geq a$, and

$$
\begin{aligned}
D_{b-}^{\alpha, \psi} f(x) & :=\left(-\frac{1}{\psi^{\prime}(x)} \frac{d}{d x}\right)^{n} I_{b-}^{n-\alpha, \psi} f(x) \\
& =\frac{1}{\Gamma(n-\alpha)}\left(-\frac{1}{\psi^{\prime}(x)} \frac{d}{d x}\right)^{n} \int_{x}^{b} \psi^{\prime}(t)(\psi(t)-\psi(x))^{n-\alpha-1} f(t) d t,
\end{aligned}
$$

for $x \leq b$, respectively.

Here, function $\psi$ is assumed to be of class $C^{n}$. In order to simplify notation, we shall write

$$
f_{\psi}^{[n]}(x):=\left(\frac{1}{\psi^{\prime}(x)} \frac{d}{d x}\right)^{n} f(x) .
$$

It is clear that, when $\alpha$ is a positive integer number, then

$$
D_{a+}^{m, \psi} f(x)=f_{\psi}^{[m]}(x) \quad \text { and } \quad D_{b-}^{m, \psi} f(x)=(-1)^{m} f_{\psi}^{[m]}(x) .
$$

This subject was extensively studied by Osler in the seventies. In particular, Osler considered various generalizations of important formulae like the Leibniz or the chain rules. Also, Osler gave generalizations of many results of the classical differential calculus like the Taylor and Laurent series. For a more detailed discussion we refer the papers $[22,23,24,25,26,27]$. 
As it was proven in [2], if $f$ is a function on class $C^{n}$, then for every $\alpha>0$,

$$
\begin{aligned}
D_{a+}^{\alpha, \psi} f(x)=\frac{1}{\Gamma(n-\alpha)} \int_{a}^{x} & \psi^{\prime}(t)(\psi(x)-\psi(t))^{n-\alpha-1} f_{\psi}^{[n]}(t) d t \\
& +\sum_{k=0}^{n-1} \frac{f_{\psi}^{[k]}(a)}{\Gamma(k+1-\alpha)}(\psi(x)-\psi(a))^{k-\alpha}
\end{aligned}
$$

and

$$
\begin{aligned}
D_{b-}^{\alpha, \psi} f(x)=\frac{1}{\Gamma(n-\alpha)} \int_{x}^{b}( & -1)^{n} \psi^{\prime}(t)(\psi(t)-\psi(x))^{n-\alpha-1} f_{\psi}^{[n]}(t) d t \\
& +\sum_{k=0}^{n-1} \frac{(-1)^{k} f_{\psi}^{[k]}(b)}{\Gamma(k+1-\alpha)}(\psi(b)-\psi(x))^{k-\alpha}
\end{aligned}
$$

These new fractional operators are called left and right Caputo fractional derivatives of $f$ of order $\alpha$, with respect to function $\psi$, and are denoted by the symbols ${ }^{C} D_{a+}^{\alpha, \psi}$ and ${ }^{C} D_{b-}^{\alpha, \psi}$ respectively:

$$
{ }^{C} D_{a+}^{\alpha, \psi} f(x)=\frac{1}{\Gamma(n-\alpha)} \int_{a}^{x} \psi^{\prime}(t)(\psi(x)-\psi(t))^{n-\alpha-1} f_{\psi}^{[n]}(t) d t
$$

and

$$
{ }^{C} D_{b-}^{\alpha, \psi} f(x)=\frac{1}{\Gamma(n-\alpha)} \int_{x}^{b}(-1)^{n} \psi^{\prime}(t)(\psi(t)-\psi(x))^{n-\alpha-1} f_{\psi}^{[n]}(t) d t .
$$

Since we are interested in studying the fractional case, we assume from now on that $\alpha \notin \mathbb{N}$.

The remainder of this paper is organized as follows. In Section 2, some properties of fractional integrals are studied, and in Section 3, some properties of fractional derivatives are also studied. In the following Section 4, a relation between these fractional operators is given. In Section 5, we present an approximation formula for each fractional operator, involving integer order derivatives only.

2. Properties of the fractional integrals. In this section, we deduce some properties for fractional integrals, like the Leibniz rule and the Taylor's theorem, and also determine the fractional integrals of some fundamental functions. Since there are two types of fractional integrals (left and right), and the proofs are similar, we will state without proof 
the results for the right fractional integrals. From now on, given a real $\alpha>0$, we assume that function $\psi$ is at least of class $C^{n}$.

Lemma 1. For every $\lambda \neq 0$ and $\alpha>0$,

$$
I_{a+}^{\alpha, \psi} E_{\alpha}\left(\lambda(\psi(x)-\psi(a))^{\alpha}\right)=\frac{1}{\lambda}\left(E_{\alpha}\left(\lambda(\psi(x)-\psi(a))^{\alpha}\right)-1\right)
$$

and

$$
I_{b-}^{\alpha, \psi} E_{\alpha}\left(\lambda(\psi(b)-\psi(x))^{\alpha}\right)=\frac{1}{\lambda}\left(E_{\alpha}\left(\lambda(\psi(b)-\psi(x))^{\alpha}\right)-1\right) .
$$

Proof. Using formula (1), we deduce the following

$$
\begin{aligned}
I_{a+}^{\alpha, \psi} E_{\alpha}\left(\lambda(\psi(x)-\psi(a))^{\alpha}\right) & =I_{a+}^{\alpha, \psi} \sum_{k=0}^{\infty} \frac{\lambda^{k}(\psi(x)-\psi(a))^{k \alpha}}{\Gamma(k \alpha+1)} \\
& =\sum_{k=0}^{\infty} \frac{\lambda^{k}(\psi(x)-\psi(a))^{k \alpha+\alpha}}{\Gamma(k \alpha+\alpha+1)} \\
& =\frac{1}{\lambda}\left(E_{\alpha}\left(\lambda(\psi(x)-\psi(a))^{\alpha}\right)-1\right) .
\end{aligned}
$$

For our next example, we recall the lower incomplete gamma function $\gamma$ :

$$
\gamma(v, z):=\int_{0}^{z} t^{v-1} \exp (-t) d t, \quad v>0 .
$$

Lemma 2. For every $\lambda \neq 0$ and $\alpha>0$,

$$
I_{a+}^{\alpha, \psi} \exp (\lambda(\psi(x)-\psi(a)))=\frac{\exp (\lambda(\psi(x)-\psi(a)))}{\lambda^{\alpha} \Gamma(\alpha)} \gamma(\alpha, \lambda(\psi(x)-\psi(a)))
$$

and

$$
I_{b-}^{\alpha, \psi} \exp (\lambda(\psi(b)-\psi(x)))=\frac{\exp (\lambda(\psi(b)-\psi(x)))}{\lambda^{\alpha} \Gamma(\alpha)} \gamma(\alpha, \lambda(\psi(b)-\psi(x))) .
$$

Proof. By definition,

$$
\begin{aligned}
& I_{a+}^{\alpha, \psi} \exp (\lambda(\psi(x)-\psi(a))) \\
& \quad=\frac{1}{\Gamma(\alpha)} \int_{a}^{x} \psi^{\prime}(t)(\psi(x)-\psi(t))^{\alpha-1} \exp (\lambda(\psi(t)-\psi(a))) d t .
\end{aligned}
$$


Making the changing of variables $\lambda(\psi(x)-\psi(t))=u$, we obtain the desired formula.

Theorem 3. Assume that $f$ has continuous derivative for $t \in[a, b]$. Then, given two differentiable functions $f$ and $\psi$,

$$
\lim _{\alpha \rightarrow 0^{+}} I_{a+}^{\alpha, \psi} f(x)=f(x) \quad \text { and } \quad \lim _{\alpha \rightarrow 0^{+}} I_{b-}^{\alpha, \psi} f(x)=f(x) .
$$

Proof. Using integration by parts, we obtain

$$
I_{a+}^{\alpha, \psi} f(x)=\frac{1}{\Gamma(\alpha+1)}\left[(\psi(x)-\psi(a))^{\alpha} f(a)+\int_{a}^{x}(\psi(x)-\psi(t))^{\alpha} f^{\prime}(t) d t\right],
$$

and making $\alpha \rightarrow 0^{+}$, the proof ends.

Theorem 4. Let $\left(f_{k}\right)$ be a sequence of continuous functions on $[a, b]$. If $\left(f_{k}\right)$ converges uniformly to a function $f$, then, for all $x \in[a, b]$, we have the following:

$$
I_{a+}^{\alpha, \psi} f(x)=\lim _{k \rightarrow \infty} I_{a+}^{\alpha, \psi} f_{k}(x) \quad \text { and } \quad I_{b-}^{\alpha, \psi} f(x)=\lim _{k \rightarrow \infty} I_{b-}^{\alpha, \psi} f_{k}(x) .
$$

Proof. Observe that $f$ is a continuous function on $[a, b]$. Then,

$$
\begin{aligned}
\left|I_{a+}^{\alpha, \psi} f(x)-I_{a+}^{\alpha, \psi} f_{k}(x)\right| & \leq \frac{1}{\Gamma(\alpha)} \int_{a}^{x} \psi^{\prime}(t)(\psi(x)-\psi(t))^{\alpha-1}\left|f(t)-f_{k}(t)\right| d t \\
& \leq \frac{(\psi(x)-\psi(a))^{\alpha}}{\Gamma(\alpha+1)}\left\|f-f_{k}\right\|,
\end{aligned}
$$

which converges to zero as $k$ goes to infinity.

Theorem 5. If $f$ is a continuous function on $[a, b]$, then $I_{a+}^{\alpha, \psi} f(x)$ and $I_{b-}^{\alpha, \psi} f(x)$ are well defined for every $x \in[a, b]$. Moreover,

$$
\left\|I_{a+}^{\alpha, \psi} f\right\| \leq K\|f\| \quad \text { and } \quad\left\|I_{b-}^{\alpha, \psi} f\right\| \leq K\|f\|,
$$

where $K=(\psi(b)-\psi(a))^{\alpha} / \Gamma(\alpha+1)$. 
Proof. Given any $x \in[a, b]$, we have that

$$
\begin{aligned}
\left|I_{a+}^{\alpha, \psi} f(x)\right| & \leq \frac{\|f\|}{\Gamma(\alpha)} \int_{a}^{x} \psi^{\prime}(t)(\psi(x)-\psi(t))^{\alpha-1} d t \\
& \leq \frac{(\psi(x)-\psi(a))^{\alpha}}{\Gamma(\alpha+1)}\|f\| \leq \frac{(\psi(b)-\psi(a))^{\alpha}}{\Gamma(\alpha+1)}\|f\| .
\end{aligned}
$$

From the proof of Theorem 5 , we see that if $f$ is a continuous function, then

$$
I_{a+}^{\alpha, \psi} f(a)=I_{b-}^{\alpha, \psi} f(b)=0 .
$$

If $f$ is not continuous, then (5) may fail. In fact, using formula (1), we see that

$$
\lim _{x \rightarrow a^{+}} I_{a+}^{\alpha, \psi}(\psi(x)-\psi(a))^{\beta-1}= \begin{cases}\infty & \text { if } \beta+\alpha-1<0 \\ \Gamma(1-\alpha) & \text { if } \beta+\alpha-1=0 \\ 0 & \text { if } \beta+\alpha-1>0\end{cases}
$$

Theorem 6. If $f$ is continuous, then, for every positive integer $m$, we have

$$
\left(\frac{1}{\psi^{\prime}(x)} \frac{d}{d x}\right)^{m} I_{a+}^{m, \psi} f(x)=\left(-\frac{1}{\psi^{\prime}(x)} \frac{d}{d x}\right)^{m} I_{b-}^{m, \psi} f(x)=f(x) .
$$

Proof. Differentiating the fractional integral, we obtain

$$
\begin{aligned}
\left(\frac{1}{\psi^{\prime}(x)} \frac{d}{d x}\right)^{m} I_{a+}^{m, \psi} f(x) & \\
& =\left(\frac{1}{\psi^{\prime}(x)} \frac{d}{d x}\right)^{m-1} \frac{1}{(m-2) !} \int_{a}^{x} \psi^{\prime}(t)(\psi(x)-\psi(t))^{m-2} f(t) d t \\
& =\left(\frac{1}{\psi^{\prime}(x)} \frac{d}{d x}\right)^{m-2} \frac{1}{(m-3) !} \int_{a}^{x} \psi^{\prime}(t)(\psi(x)-\psi(t))^{m-3} f(t) d t \\
& =\cdots=\frac{1}{\psi^{\prime}(x)} \frac{d}{d x} \int_{a}^{x} \psi^{\prime}(t) f(t) d t=f(x) .
\end{aligned}
$$

Corollary 7. If $f$ is continuous, then, for every positive integer $m$ and every real $\alpha>m$, we have

$$
\left(\frac{1}{\psi^{\prime}(x)} \frac{d}{d x}\right)^{m} I_{a+}^{\alpha, \psi} f(x)=I_{a+}^{\alpha-m, \psi} f(x)
$$


and

$$
\left(-\frac{1}{\psi^{\prime}(x)} \frac{d}{d x}\right)^{m} I_{b-}^{\alpha, \psi} f(x)=I_{b-}^{\alpha-m, \psi} f(x) .
$$

Theorem 8. If $f$ is a function of class $C^{m}$, with $m \in \mathbb{N}$, then

and

$$
I_{a+}^{m, \psi}\left(\frac{1}{\psi^{\prime}(x)} \frac{d}{d x}\right)^{m} f(x)=f(x)-\sum_{k=0}^{m-1} \frac{f^{[k]}(a)}{k !}(\psi(x)-\psi(a))^{k}
$$

$$
I_{b-}^{m, \psi}\left(-\frac{1}{\psi^{\prime}(x)} \frac{d}{d x}\right)^{m} f(x)=f(x)-\sum_{k=0}^{m-1} \frac{(-1)^{k} f^{[k]}(b)}{k !}(\psi(b)-\psi(x))^{k} .
$$

Proof. Integrating by parts, we get

$$
\begin{aligned}
& I_{a+}^{m, \psi}\left(\frac{1}{\psi^{\prime}(x)} \frac{d}{d x}\right)^{m} f(x) \\
& =\frac{1}{(m-1) !} \int_{a}^{x}(\psi(x)-\psi(t))^{m-1} \frac{d}{d t}\left[\left(\frac{1}{\psi^{\prime}(t)} \frac{d}{d t}\right)^{m-1} f(t)\right] d t \\
& =\frac{1}{(m-2) !} \int_{a}^{x}(\psi(x)-\psi(t))^{m-2} \frac{d}{d t}\left[\left(\frac{1}{\psi^{\prime}(t)} \frac{d}{d t}\right)^{m-2} f(t)\right] d t \\
& -\frac{f^{[m-1]}(a)}{(m-1) !}(\psi(x)-\psi(a))^{m-1} \\
& =\frac{1}{(m-3) !} \int_{a}^{x}(\psi(x)-\psi(t))^{m-3} \frac{d}{d t}\left[\left(\frac{1}{\psi^{\prime}(t)} \frac{d}{d t}\right)^{m-3} f(t)\right] d t \\
& -\sum_{k=m-2}^{m-1} \frac{f^{[k]}(a)}{k !}(\psi(x)-\psi(a))^{k} \\
& =\cdots=\int_{a}^{x} \frac{d}{d t} f(t) d t-\sum_{k=1}^{m-1} \frac{f^{[k]}(a)}{k !}(\psi(x)-\psi(a))^{k} \\
& =f(x)-\sum_{k=0}^{m-1} \frac{f^{[k]}(a)}{k !}(\psi(x)-\psi(a))^{k} .
\end{aligned}
$$

Corollary 9. If $f$ is a function of class $C^{m}$, with $m \in \mathbb{N}$, then

$$
I_{a+}^{\alpha+m, \psi}\left(\frac{1}{\psi^{\prime}(x)} \frac{d}{d x}\right)^{m} f(x)=I_{a+}^{\alpha, \psi} f(x)-\sum_{k=0}^{m-1} \frac{f^{[k]}(a)}{\Gamma(\alpha+k+1)}(\psi(x)-\psi(a))^{\alpha+k}
$$


and

$$
\begin{aligned}
& I_{b-}^{\alpha+m, \psi}\left(-\frac{1}{\psi^{\prime}(x)} \frac{d}{d x}\right)^{m} \\
& \quad f(x) \\
& \quad=I_{b-}^{\alpha, \psi} f(x)-\sum_{k=0}^{m-1} \frac{(-1)^{k} f^{[k]}(b)}{\Gamma(\alpha+k+1)}(\psi(b)-\psi(x))^{\alpha+k} .
\end{aligned}
$$

Proof. By the semigroup law and Theorem 8, we obtain

$$
\begin{aligned}
I_{a+}^{\alpha+m, \psi}\left(\frac{1}{\psi^{\prime}(x)} \frac{d}{d x}\right)^{m} f(x) & \\
& =I_{a+}^{\alpha, \psi}\left(I_{a+}^{m, \psi}\left(\frac{1}{\psi^{\prime}(x)} \frac{d}{d x}\right)^{m} f(x)\right) \\
& =I_{a+}^{\alpha, \psi}\left(f(x)-\sum_{k=0}^{m-1} \frac{f^{[k]}(a)}{k !}(\psi(x)-\psi(a))^{k}\right) \\
& =I_{a+}^{\alpha, \psi} f(x)-\sum_{k=0}^{m-1} \frac{f^{[k]}(a)}{\Gamma(\alpha+k+1)}(\psi(x)-\psi(a))^{\alpha+k}
\end{aligned}
$$

Theorem 10. Let $f$ and $g$ be two continuous functions on $[a, b]$. Then,

and

$$
\int_{a}^{b} f(x) I_{a+}^{\alpha, \psi} g(x) d x=\int_{a}^{b} I_{b-}^{\alpha, \psi}\left(\frac{f(x)}{\psi^{\prime}(x)}\right) g(x) \psi^{\prime}(x) d x
$$

$$
\int_{a}^{b} f(x) I_{b-}^{\alpha, \psi} g(x) d x=\int_{a}^{b} I_{a+}^{\alpha, \psi}\left(\frac{f(x)}{\psi^{\prime}(x)}\right) g(x) \psi^{\prime}(x) d x .
$$

Proof. Using the Dirichlet's formula, we deduce

$$
\begin{aligned}
\int_{a}^{b} f(x) I_{a+}^{\alpha, \psi} g(x) & d x \\
& =\frac{1}{\Gamma(\alpha)} \int_{a}^{b} \int_{a}^{x} f(x) \psi^{\prime}(t)(\psi(x)-\psi(t))^{\alpha-1} g(t) d t d x \\
& =\frac{1}{\Gamma(\alpha)} \int_{a}^{b} \int_{x}^{b} f(t) \psi^{\prime}(x)(\psi(t)-\psi(x))^{\alpha-1} g(x) d t d x \\
& =\int_{a}^{b} I_{b-}^{\alpha, \psi}\left(\frac{f(x)}{\psi^{\prime}(x)}\right) g(x) \psi^{\prime}(x) d x .
\end{aligned}
$$


Theorem 11. Suppose that function $f \circ \psi^{-1}$ is analytic on $[\psi(a), \psi(b)]$. Then, for all $x \in[a, b]$,

$$
I_{a+}^{\alpha, \psi} f(x)=\sum_{k=0}^{\infty} \frac{D^{k}\left(f \circ \psi^{-1}\right) \psi(a)}{\Gamma(k+\alpha+1)}(\psi(x)-\psi(a))^{k+\alpha}
$$

and

$$
I_{b-}^{\alpha, \psi} f(x)=\sum_{k=0}^{\infty} \frac{(-1)^{k} D^{k}\left(f \circ \psi^{-1}\right) \psi(b)}{\Gamma(k+\alpha+1)}(\psi(b)-\psi(x))^{k+\alpha} .
$$

Proof. Once $f \circ \psi^{-1}$ is analytic, we can consider the series

$$
f(x)=\left(f \circ \psi^{-1}\right)(\psi(x))=\sum_{k=0}^{\infty} \frac{D^{k}\left(f \circ \psi^{-1}\right) \psi(a)}{k !}(\psi(x)-\psi(a))^{k} .
$$

Applying formula (1), we deduce the desired result.

Theorem 12. Suppose that function $f \circ \psi^{-1}$ is analytic on $[\psi(a), \psi(b)]$. Then, for all $x \in[a, b]$,

$$
I_{a+}^{\alpha, \psi} f(x)=\sum_{k=0}^{\infty}\left(\begin{array}{r}
-\alpha \\
k
\end{array}\right) \frac{D^{k}\left(f \circ \psi^{-1}\right) \psi(x)}{\Gamma(k+\alpha+1)}(\psi(x)-\psi(a))^{k+\alpha}
$$

and

$$
I_{b-}^{\alpha, \psi} f(x)=\sum_{k=0}^{\infty}(-1)^{k}\left(\begin{array}{r}
-\alpha \\
k
\end{array}\right) \frac{D^{k}\left(f \circ \psi^{-1}\right) \psi(x)}{\Gamma(k+\alpha+1)}(\psi(b)-\psi(x))^{k+\alpha},
$$

where

$$
\left(\begin{array}{r}
-\alpha \\
k
\end{array}\right)=\frac{(-1)^{k} \Gamma(k+\alpha)}{\Gamma(\alpha) k !} .
$$

Proof. By the definition of fractional integral,

$$
\begin{aligned}
I_{a+}^{\alpha, \psi} f(x)= & \frac{1}{\Gamma(\alpha)} \int_{a}^{x} \psi^{\prime}(t)(\psi(x)-\psi(t))^{\alpha-1}\left(f \circ \psi^{-1}\right) \psi(t) d t \\
= & \frac{1}{\Gamma(\alpha)} \int_{a}^{x} \psi^{\prime}(t)(\psi(x)-\psi(t))^{\alpha-1} \\
& \quad \times \sum_{k=0}^{\infty} \frac{(-1)^{k} D^{k}\left(f \circ \psi^{-1}\right) \psi(x)}{k !}(\psi(x)-\psi(t))^{k} d t
\end{aligned}
$$




$$
\begin{aligned}
& =\sum_{k=0}^{\infty} \frac{(-1)^{k} D^{k}\left(f \circ \psi^{-1}\right) \psi(x)}{\Gamma(\alpha) k !} \\
& \quad \times \int_{a}^{x} \psi^{\prime}(t)(\psi(x)-\psi(t))^{k+\alpha-1} d t \\
& =\sum_{k=0}^{\infty}\left(\begin{array}{r}
-\alpha \\
k
\end{array}\right) \frac{D^{k}\left(f \circ \psi^{-1}\right) \psi(x)}{\Gamma(k+\alpha+1)}(\psi(x)-\psi(a))^{k+\alpha} .
\end{aligned}
$$

Theorem 13. Let $f, g:[a, b] \rightarrow \mathbb{R}$ be two functions with

(1) $f \circ \psi^{-1}$ is analytic at $y$ for all $y \in[\psi(a), \psi(b)]$, and

(2) $g$ is continuous on $[a, b]$.

Then, for $x \in[a, b]$,

$$
I_{a+}^{\alpha, \psi}(f \cdot g)(x)=\sum_{k=0}^{\infty}\left(\begin{array}{r}
-\alpha \\
k
\end{array}\right) D^{k}\left(f \circ \psi^{-1}\right) \psi(x) \cdot I_{a+}^{\alpha+k, \psi} g(x)
$$

and

$$
I_{b-}^{\alpha, \psi}(f \cdot g)(x)=\sum_{k=0}^{\infty}(-1)^{k}\left(\begin{array}{r}
-\alpha \\
k
\end{array}\right) D^{k}\left(f \circ \psi^{-1}\right) \psi(x) \cdot I_{b-}^{\alpha+k, \psi} g(x) .
$$

Proof. Once $f \circ \psi^{-1}$ is analytic,

$$
f(t)=\sum_{k=0}^{\infty} \frac{(-1)^{k} D^{k}\left(f \circ \psi^{-1}\right) \psi(x)}{k !}(\psi(x)-\psi(t))^{k} .
$$

Therefore,

$$
\begin{aligned}
I_{a+}^{\alpha, \psi}(f \cdot g)(x) & =\sum_{k=0}^{\infty} \frac{(-1)^{k} D^{k}\left(f \circ \psi^{-1}\right) \psi(x)}{k ! \Gamma(\alpha)} \\
& \times \int_{a}^{x} \psi^{\prime}(t)(\psi(x)-\psi(t))^{\alpha+k-1} g(t) d t \\
& =\sum_{k=0}^{\infty} \frac{(-1)^{k} \Gamma(\alpha+k) D^{k}\left(f \circ \psi^{-1}\right) \psi(x)}{k ! \Gamma(\alpha)} \cdot I_{a+}^{\alpha+k, \psi} g(x) \\
& =\sum_{k=0}^{\infty}\left(\begin{array}{r}
-\alpha \\
k
\end{array}\right) D^{k}\left(f \circ \psi^{-1}\right) \psi(x) \cdot I_{a+}^{\alpha+k, \psi} g(x) .
\end{aligned}
$$




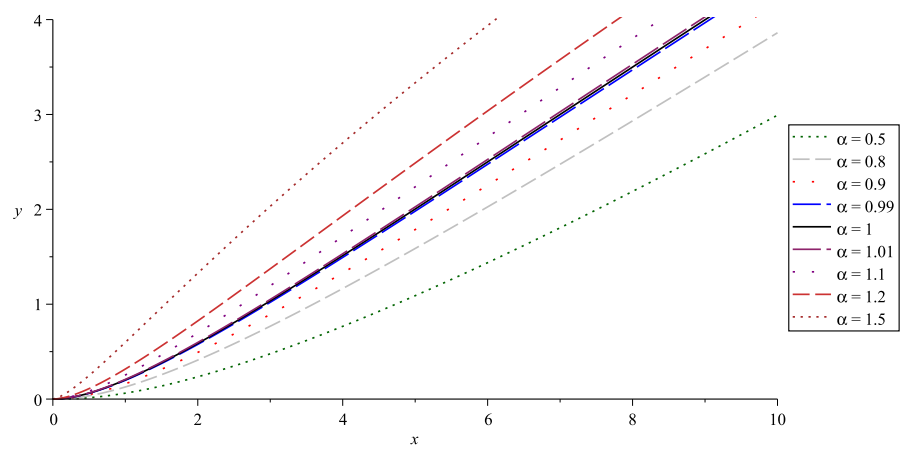

Figure 1. Fractional derivative of $f(x)=(\psi(x)-\psi(0))^{3}$.

3. Properties of the fractional derivatives. We proceed our study by considering now the fractional derivative operator. First, we evaluate the fractional derivative of some important functions. Then, we study in which cases we can use the semigroup law for fractional derivatives. Other properties are also considered, like an integration by parts formula involving these fractional operators.

Lemma 14. (See [16, Property 2.20]) For any $\beta>0$, the two following formulas hold:

and

$$
D_{a+}^{\alpha, \psi}(\psi(x)-\psi(a))^{\beta-1}=\frac{\Gamma(\beta)}{\Gamma(\beta-\alpha)}(\psi(x)-\psi(a))^{\beta-\alpha-1}
$$

$$
D_{b-}^{\alpha, \psi}(\psi(b)-\psi(x))^{\beta-1}=\frac{\Gamma(\beta)}{\Gamma(\beta-\alpha)}(\psi(b)-\psi(x))^{\beta-\alpha-1} .
$$

For example, in Figure 1, we plot the graphs of the fractional derivative of order $\alpha$ of function $f(x)=(\psi(x)-\psi(0))^{3}$, with $x \in[0,10]$. The kernel is the function $\psi(x)=\sqrt[3]{x+1}$. As it can be observed, as $\alpha$ goes to 1 , the graph of the fractional derivative $D_{0+}^{\alpha, \psi} f(x)$ approaches the graph of $f_{\psi}^{[1]}=3(\psi(x)-\psi(0))^{2}$.

Lemma 15. For every $\lambda \in \mathbb{R}$ and $\alpha>0(\alpha \notin \mathbb{N})$,

$D_{a+}^{\alpha, \psi} E_{\alpha}\left(\lambda(\psi(x)-\psi(a))^{\alpha}\right)=\lambda E_{\alpha}\left(\lambda(\psi(x)-\psi(a))^{\alpha}\right)+\frac{(\psi(x)-\psi(a))^{-\alpha}}{\Gamma(1-\alpha)}$ 
and $D_{b-}^{\alpha, \psi} E_{\alpha}\left(\lambda(\psi(b)-\psi(x))^{\alpha}\right)=\lambda E_{\alpha}\left(\lambda(\psi(b)-\psi(x))^{\alpha}\right)+\frac{(\psi(b)-\psi(x))^{-\alpha}}{\Gamma(1-\alpha)}$.

Proof. Using Lemma 14, we obtain

$$
\begin{aligned}
D_{a+}^{\alpha, \psi} E_{\alpha}(\lambda(\psi(x)-\psi & \left.(a))^{\alpha}\right) \\
& =\sum_{k=0}^{\infty} \frac{\lambda^{k}}{\Gamma(k \alpha+1)} D_{a+}^{\alpha, \psi}(\psi(x)-\psi(a))^{k \alpha} \\
& =\sum_{k=0}^{\infty} \frac{\lambda^{k}(\psi(x)-\psi(a))^{k \alpha-\alpha}}{\Gamma(k \alpha-\alpha+1)} \\
& =\lambda \sum_{k=0}^{\infty} \frac{\lambda^{k}(\psi(x)-\psi(a))^{k \alpha}}{\Gamma(k \alpha+1)}+\frac{(\psi(x)-\psi(a))^{-\alpha}}{\Gamma(1-\alpha)} \\
& =\lambda E_{\alpha}\left(\lambda(\psi(x)-\psi(a))^{\alpha}\right)+\frac{(\psi(x)-\psi(a))^{-\alpha}}{\Gamma(1-\alpha)} .
\end{aligned}
$$

Lemma 16. For every $\lambda \neq 0$ and $\alpha>0(\alpha \notin \mathbb{N})$, $D_{a+}^{\alpha, \psi} \exp (\lambda(\psi(x)-\psi(a)))=\frac{\exp (\lambda(\psi(x)-\psi(a)))}{\lambda^{-\alpha} \Gamma(-\alpha)} \gamma(-\alpha, \lambda(\psi(x)-\psi(a)))$ and $D_{b-}^{\alpha, \psi} \exp (\lambda(\psi(b)-\psi(x)))=\frac{\exp (\lambda(\psi(b)-\psi(x)))}{\lambda^{-\alpha} \Gamma(-\alpha)} \gamma(-\alpha, \lambda(\psi(b)-\psi(x)))$.

Proof. Integrating by parts and differentiating the result, we obtain

$$
\begin{aligned}
& D_{a+}^{\alpha, \psi} \exp (\lambda(\psi(x)-\psi(a))) \\
& =\left(\frac{1}{\psi^{\prime}(x)} \frac{d}{d x}\right)^{n}\left(\frac { 1 } { \Gamma ( n - \alpha ) } \left[\frac{(\psi(x)-\psi(a))^{n-\alpha}}{n-\alpha}\right.\right. \\
& +\int_{a}^{x} \lambda \psi^{\prime}(t) \frac{(\psi(x)-\psi(t))^{n-\alpha}}{n-\alpha} \\
& \quad \times \exp (\lambda(\psi(t)-\psi(a))) d t])
\end{aligned}
$$




$$
\begin{aligned}
&=\left(\frac{1}{\psi^{\prime}(x)} \frac{d}{d x}\right)^{n-1}( \frac{(\psi(x)-\psi(a))^{n-\alpha-1}}{\Gamma(n-\alpha)} \\
&+\frac{\lambda}{\Gamma(n-\alpha)} \int_{a}^{x} \psi^{\prime}(t)(\psi(x)-\psi(t))^{n-\alpha-1} \\
&\quad \times \exp (\lambda(\psi(t)-\psi(a))) d t)=\star
\end{aligned}
$$

Integrating again by parts,

$$
\begin{aligned}
\star & \left(\frac{1}{\psi^{\prime}(x)} \frac{d}{d x}\right)^{n-1} \\
& \left(\frac{(\psi(x)-\psi(a))^{n-\alpha-1}}{\Gamma(n-\alpha)}+\frac{\lambda}{\Gamma(n-\alpha)}\left[\frac{(\psi(x)-\psi(a))^{n-\alpha}}{n-\alpha}\right.\right. \\
& \left.\left.+\int_{a}^{x} \lambda \psi^{\prime}(t) \frac{(\psi(x)-\psi(t))^{n-\alpha}}{n-\alpha} \exp (\lambda(\psi(t)-\psi(a))) d t\right]\right) \\
= & \left.\left(\frac{1}{\psi^{\prime}(x)} \frac{d}{d x}\right)^{n-2}\right] \\
& \left(\frac{(\psi(x)-\psi(a))^{n-\alpha-2}}{\Gamma(n-\alpha-1)}+\lambda \frac{(\psi(x)-\psi(a))^{n-\alpha-1}}{\Gamma(n-\alpha)}\right. \\
& \left.+\frac{\lambda^{2}}{\Gamma(n-\alpha)} \int_{a}^{x} \psi^{\prime}(t)(\psi(x)-\psi(t))^{n-\alpha-1} \exp (\lambda(\psi(t)-\psi(a))) d t\right) .
\end{aligned}
$$

Repeating this procedure, we conclude that

$$
\begin{aligned}
\star & =\sum_{k=0}^{n-1} \lambda^{k} \frac{(\psi(x)-\psi(a))^{k-\alpha}}{\Gamma(k-\alpha+1)} \\
& \quad+\frac{\lambda^{n}}{\Gamma(n-\alpha)} \int_{a}^{x} \psi^{\prime}(t)(\psi(x)-\psi(t))^{n-\alpha-1} \exp (\lambda(\psi(t)-\psi(a))) d t .
\end{aligned}
$$

In a similar way as was done in the proof of Lemma 2, we can prove

$$
\begin{aligned}
\frac{\lambda^{n}}{\Gamma(n-\alpha)} \int_{a}^{x} \psi^{\prime}(t)(\psi(x) & -\psi(t))^{n-\alpha-1} \exp (\lambda(\psi(t)-\psi(a))) d t \\
= & \frac{\exp (\lambda(\psi(x)-\psi(a)))}{\lambda^{-\alpha} \Gamma(n-\alpha)} \gamma(n-\alpha, \lambda(\psi(x)-\psi(a))) .
\end{aligned}
$$




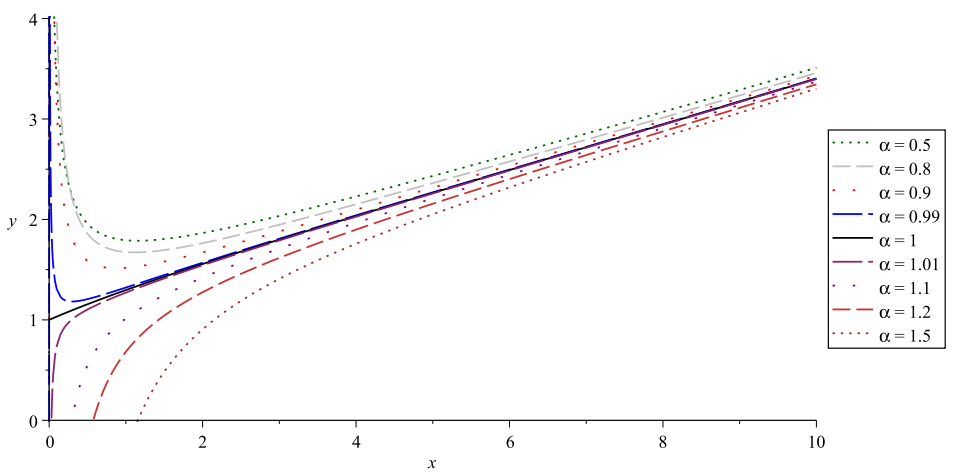

FIgURE 2. Fractional derivative of $f(x)=\exp (\psi(x)-\psi(0))$.

Using the recurrence relation for the lower incomplete gamma function (see [33, Equation (1.4)])

$$
\gamma(s+1, z)=s \gamma(s, z)-z^{s} \exp (-z),
$$

we deduce that

$$
\begin{array}{r}
\frac{\exp (\lambda(\psi(x)-\psi(a)))}{\lambda^{-\alpha} \Gamma(n-\alpha)} \gamma(n-\alpha, \lambda(\psi(x)-\psi(a))) \\
=\frac{\exp (\lambda(\psi(x)-\psi(a)))}{\lambda^{-\alpha} \Gamma(-\alpha)} \gamma(-\alpha, \lambda(\psi(x)-\psi(a))) \\
-\sum_{k=0}^{n-1} \lambda^{k} \frac{(\psi(x)-\psi(a))^{k-\alpha}}{\Gamma(k-\alpha+1)} .
\end{array}
$$

Therefore, we conclude that

$$
\begin{aligned}
D_{a+}^{\alpha, \psi} \exp (\lambda(\psi(x) & -\psi(a))) \\
& =\frac{\exp (\lambda(\psi(x)-\psi(a)))}{\lambda^{-\alpha} \Gamma(-\alpha)} \gamma(-\alpha, \lambda(\psi(x)-\psi(a))) .
\end{aligned}
$$

In Figure 2 we show the graphs of the fractional derivatives of the function $f(x)=\exp (\psi(x)-\psi(0))$, with $x \in[0,10]$, with respect to the kernel $\psi(x)=\sqrt[3]{x+1}$, for different values of $\alpha$. 
Theorem 17. Assume that $f \in C^{n}[a, b]$. Then,

$$
\lim _{\alpha \rightarrow(n-1)^{+}} D_{a+}^{\alpha, \psi} f(x)=f_{\psi}^{[n-1]}(x)
$$

and

$$
\lim _{\alpha \rightarrow(n-1)^{+}} D_{b-}^{\alpha, \psi} f(x)=(-1)^{n-1} f_{\psi}^{[n-1]}(x) .
$$

In addition, if $f^{(n)}$ is continuously differentiable, then

$$
\lim _{\alpha \rightarrow n^{-}} D_{a+}^{\alpha, \psi} f(x)=f_{\psi}^{[n]}(x)
$$

and

$$
\lim _{\alpha \rightarrow n^{-}} D_{b-}^{\alpha, \psi} f(x)=(-1)^{n} f_{\psi}^{[n]}(x) .
$$

Proof. Using formula (3), we deduce that

$$
\begin{gathered}
\lim _{\alpha \rightarrow(n-1)^{+}} D_{a+}^{\alpha, \psi} f(x) \\
=\lim _{\alpha \rightarrow(n-1)^{+}}\left(\frac{1}{\Gamma(n-\alpha)} \int_{a}^{x} \psi^{\prime}(t)(\psi(x)-\psi(t))^{n-\alpha-1} f_{\psi}^{[n]}(t) d t\right. \\
\left.\quad+\sum_{k=0}^{n-1} \frac{f_{\psi}^{[k]}(a)}{\Gamma(k+1-\alpha)}(\psi(x)-\psi(a))^{k-\alpha}\right) \\
=\int_{a}^{x} \psi^{\prime}(t) f_{\psi}^{[n]}(t) d t+f_{\psi}^{[n-1]}(a)=f_{\psi}^{[n-1]}(x),
\end{gathered}
$$

since $\Gamma(k)=\infty$, for $k \in \mathbb{Z}^{-} \cup\{0\}$. Suppose now that $f \in C^{n+1}[a, b]$. Integrating by parts, we get

$$
\begin{aligned}
\frac{1}{\Gamma(n-\alpha)} \int_{a}^{x} \psi^{\prime}(t)(\psi(x)-\psi(t))^{n-\alpha-1} f_{\psi}^{[n]}(t) d t \\
=\frac{f_{\psi}^{[n]}(a)}{\Gamma(n-\alpha+1)}(\psi(x)-\psi(a))^{n-\alpha} \\
\quad+\frac{1}{\Gamma(n-\alpha+1)} \int_{a}^{x}(\psi(x)-\psi(t))^{n-\alpha} \frac{d}{d t} f_{\psi}^{[n]}(t) d t,
\end{aligned}
$$

and so

$$
\lim _{\alpha \rightarrow n^{-}} D_{a+}^{\alpha, \psi} f(x)=f_{\psi}^{[n]}(a)+\int_{a}^{x} \frac{d}{d t} f_{\psi}^{[n]}(t) d t=f_{\psi}^{[n]}(x) .
$$


Theorem 18. Suppose that function $f \circ \psi^{-1}$ is analytic in a neighborhood of $\psi(a)$ (resp. $\psi(b))$. Then,

(1) $\lim _{x \rightarrow a^{+}} D_{a+}^{\alpha, \psi} f(x)=\infty$ unless $f(a)=0$ and $D^{k}\left(f \circ \psi^{-1}\right) \psi(a)=0$, for every $k=1, \ldots, n-1$.

(2) $\lim _{x \rightarrow b^{-}} D_{b-}^{\alpha, \psi} f(x)=\infty$ unless $f(b)=0$ and $D^{k}\left(f \circ \psi^{-1}\right) \psi(b)=0$, for every $k=1, \ldots, n-1$.

Proof. Since $f \circ \psi^{-1}$ is analytic,

$$
f(x)=\sum_{k=0}^{\infty} \frac{D^{k}\left(f \circ \psi^{-1}\right) \psi(a)}{k !}(\psi(x)-\psi(a))^{k},
$$

and so

$$
D_{a+}^{\alpha, \psi} f(x)=\sum_{k=0}^{\infty} \frac{D^{k}\left(f \circ \psi^{-1}\right) \psi(a)}{\Gamma(k+1-\alpha)}(\psi(x)-\psi(a))^{k-\alpha},
$$

proving the desired result.

Theorem 19. Let $\left(f_{k}\right)$ be a sequence of continuous functions on $[a, b]$ such that $D_{a+}^{\alpha, \psi} f_{k}$ exists for all $k \in \mathbb{N}$. If $\left(f_{k}\right)$ converges uniformly to a function $f$ and $\left(D_{a+}^{\alpha, \psi} f_{k}\right)$ converges uniformly on $[a+\epsilon, b]$, for all $0<\epsilon<b-a$, then

$$
D_{a+}^{\alpha, \psi} f(x)=\lim _{k \rightarrow \infty} D_{a+}^{\alpha, \psi} f_{k}(x), \quad x>a .
$$

Also, if $D_{b-}^{\alpha, \psi} f_{k}$ exists for all $k \in \mathbb{N}$ and $\left(D_{b-}^{\alpha, \psi} f_{k}\right)$ converges uniformly on $[a, b-\epsilon]$, for all $0<\epsilon<b-a$, then

$$
D_{b-}^{\alpha, \psi} f(x)=\lim _{k \rightarrow \infty} D_{b-}^{\alpha, \psi} f_{k}(x), \quad x<b .
$$

Proof. By Theorem 4,

$$
I_{a+}^{n-\alpha, \psi} f(x)=\lim _{k \rightarrow \infty} I_{a+}^{n-\alpha, \psi} f_{k}(x) .
$$

On the other hand, since

$$
D_{a+}^{\alpha, \psi} f_{k}(x)=\left(\frac{1}{\psi^{\prime}(x)} \frac{d}{d x}\right)^{n} I_{a+}^{n-\alpha, \psi} f_{k}(x)
$$


and $\left(D_{a+}^{\alpha, \psi} f_{k}\right)$ converges uniformly on every compact subinterval of $(a, b]$, we may interchange the limit operator and the differential operator, concluding the desired formula.

Theorem 20. Suppose that $f \in C^{n}[a, b]$ and let

$$
K=\frac{(\psi(b)-\psi(a))^{n-\alpha}}{\Gamma(n-\alpha+1)} .
$$

If $f^{(k)}(a)=0$, for every $k=0,1, \ldots, n-1$, then $D_{a+}^{\alpha, \psi} f(x)$ is well defined for every $x \in[a, b]$ and $\left\|D_{a+}^{\alpha, \psi} f\right\| \leq K\left\|f_{\psi}^{[n]}\right\|$. If $f^{(k)}(b)=0$, for every $k=0,1, \ldots, n-1$, then $D_{b-}^{\alpha, \psi} f(x)$ is well defined and $\left\|D_{b-}^{\alpha, \psi} f\right\| \leq K\left\|f_{\psi}^{[n]}\right\|$.

Proof. Using formula (3), we deduce the result.

Observe that the condition

$$
f^{(k)}(a)=0 \text { for every } k=0,1, \ldots, n-1
$$

is essential. For example, if we consider $f(x)=E_{\alpha}\left(\lambda(\psi(x)-\psi(a))^{\alpha}\right)$, then $f \in C^{n}[a, b]$ but

$$
\lim _{x \rightarrow a^{+}} D_{a+}^{\alpha, \psi} f(x)=\infty
$$

Theorem 21. Let $f \in C^{1}[a, b]$ and $\alpha \in(0,1)$.

(1) If $f^{\prime}$ is nonnegative and $f(a) \geq 0$, then $D_{a+}^{\alpha, \psi} f$ is nonnegative.

(2) If $f^{\prime}$ is nonpositive and $f(b) \geq 0$, then $D_{b-}^{\alpha, \psi} f$ is nonnegative.

Proof. The result follows using the following formula:

$$
\begin{aligned}
& D_{a+}^{\alpha, \psi} f(x) \\
& =\frac{1}{\Gamma(1-\alpha)} \int_{a}^{x}(\psi(x)-\psi(t))^{-\alpha} f^{\prime}(t) d t+\frac{f(a)}{\Gamma(1-\alpha)}(\psi(x)-\psi(a))^{-\alpha} .
\end{aligned}
$$

Theorem 22. Let $f \in C[a, b]$ and $g \in C^{n}[a, b]$ such that $D_{a+}^{\alpha, \psi} g$ and $D_{b-}^{\alpha, \psi} g$ exist on $[a, b]$. Then,

$$
\int_{a}^{b} f(x) D_{a+}^{\alpha, \psi} g(x) d x=\int_{a}^{b} D_{b-}^{\alpha, \psi}\left(\frac{f(x)}{\psi^{\prime}(x)}\right) g(x) \psi^{\prime}(x) d x
$$


and

$$
\int_{a}^{b} f(x) D_{b-}^{\alpha, \psi} g(x) d x=\int_{a}^{b} D_{a+}^{\alpha, \psi}\left(\frac{f(x)}{\psi^{\prime}(x)}\right) g(x) \psi^{\prime}(x) d x .
$$

Proof. Since $g$ is of class $C^{n}$, then ${ }^{C} D_{a+}^{\alpha, \psi} g$ and ${ }^{C} D_{b-}^{\alpha, \psi} g$ are continuous functions on $[a, b]$ (see [2, Theorem 2]). Also, by (3) and (4), we conclude that $g_{\psi}^{[k]}(a)=g_{\psi}^{[k]}(b)=0$, for all $k=0,1, \ldots, n-1$. Therefore, by $[2$, Theorem 12$]$

$$
\begin{aligned}
\int_{a}^{b} f(x) D_{a+}^{\alpha, \psi} g(x) d x & =\int_{a}^{b} f(x)^{C} D_{a+}^{\alpha, \psi} g(x) d x \\
& =\int_{a}^{b} D_{b-}^{\alpha, \psi}\left(\frac{f(x)}{\psi^{\prime}(x)}\right) g(x) \psi^{\prime}(x) d x .
\end{aligned}
$$

Theorem 23. Suppose that function $f \circ \psi^{-1}$ is analytic on $[\psi(a), \psi(b)]$. Then, for all $x \in[a, b]$,

$$
D_{a+}^{\alpha, \psi} f(x)=\sum_{k=0}^{\infty} \frac{D^{k}\left(f \circ \psi^{-1}\right) \psi(a)}{\Gamma(k-\alpha+1)}(\psi(x)-\psi(a))^{k-\alpha}
$$

and

$$
D_{b-}^{\alpha, \psi} f(x)=\sum_{k=0}^{\infty} \frac{(-1)^{k} D^{k}\left(f \circ \psi^{-1}\right) \psi(b)}{\Gamma(k-\alpha+1)}(\psi(b)-\psi(x))^{k-\alpha} .
$$

Proof. Considering the series

$$
f(x)=\left(f \circ \psi^{-1}\right)(\psi(x))=\sum_{k=0}^{\infty} \frac{D^{k}\left(f \circ \psi^{-1}\right) \psi(a)}{k !}(\psi(x)-\psi(a))^{k}
$$

and Lemma 14, we obtain formula (6).

Theorem 24. Suppose that function $f \circ \psi^{-1}$ is analytic on $[\psi(a), \psi(b)]$. Then, for all $x \in[a, b]$,

$$
D_{a+}^{\alpha, \psi} f(x)=\sum_{k=0}^{\infty}\left(\begin{array}{l}
\alpha \\
k
\end{array}\right) \frac{D^{k}\left(f \circ \psi^{-1}\right) \psi(x)}{\Gamma(k-\alpha+1)}(\psi(x)-\psi(a))^{k-\alpha}
$$


and

$$
D_{b-}^{\alpha, \psi} f(x)=\sum_{k=0}^{\infty}(-1)^{k}\left(\begin{array}{l}
\alpha \\
k
\end{array}\right) \frac{D^{k}\left(f \circ \psi^{-1}\right) \psi(x)}{\Gamma(k-\alpha+1)}(\psi(b)-\psi(x))^{k-\alpha} .
$$

Proof. By definition of fractional derivative,

$$
\begin{aligned}
D_{a+}^{\alpha, \psi} f(x) & =\left(\frac{1}{\psi^{\prime}(x)} \frac{d}{d x}\right)^{n} I_{a+}^{n-\alpha, \psi} f(x) \\
& =\left(\frac{1}{\psi^{\prime}(x)} \frac{d}{d x}\right)^{n} \sum_{k=0}^{\infty}\left(\begin{array}{c}
\alpha-n \\
k
\end{array}\right) \frac{D^{k}\left(f \circ \psi^{-1}\right) \psi(x)}{\Gamma(k+n-\alpha+1)}(\psi(x)-\psi(a))^{k+n-\alpha} \\
& =\sum_{k=0}^{\infty}\left(\begin{array}{c}
\alpha-n \\
k
\end{array}\right) \frac{1}{\Gamma(k+n-\alpha+1)}\left(\frac{1}{\psi^{\prime}(x)} \frac{d}{d x}\right)^{n} \\
& =\sum_{k=0}^{\infty}\left(\begin{array}{c}
\alpha-n \\
k
\end{array}\right) \sum_{j=0}^{n}\left(\begin{array}{c}
n \\
j
\end{array}\right) \frac{D^{k+j}\left(f \circ \psi^{-1}\right) \psi(x)}{\Gamma(k+j-\alpha+1)}(\psi(x)-\psi(a))^{k+j-\alpha} \\
& =\sum_{k=0}^{\infty} \sum_{j=0}^{\infty}\left(\begin{array}{c}
\alpha-n \\
k
\end{array}\right)\left(\begin{array}{c}
n \\
j
\end{array}\right) \frac{D^{k+j}\left(f \circ \psi^{-1}\right) \psi(x)}{\Gamma(k+j-\alpha+1)}(\psi(x)-\psi(a))^{k+j-\alpha},
\end{aligned}
$$

since

$$
\left(\begin{array}{l}
n \\
j
\end{array}\right)=0, \quad j>n .
$$

Letting $k^{\prime}=k+j$ and using the identity [31, Equation (1.53)]

$$
\sum_{l=0}^{p}\left(\begin{array}{l}
a \\
l
\end{array}\right)\left(\begin{array}{c}
b \\
p-l
\end{array}\right)=\left(\begin{array}{c}
a+b \\
p
\end{array}\right),
$$

we obtain

$$
\begin{aligned}
D_{a+}^{\alpha, \psi} & f(x) \\
& =\sum_{k^{\prime}=0}^{\infty} \sum_{k=0}^{k^{\prime}}\left(\begin{array}{c}
\alpha-n \\
k
\end{array}\right)\left(\begin{array}{c}
n \\
k^{\prime}-k
\end{array}\right) \frac{D^{k^{\prime}}\left(f \circ \psi^{-1}\right) \psi(x)}{\Gamma\left(k^{\prime}-\alpha+1\right)}(\psi(x)-\psi(a))^{k^{\prime}-\alpha} \\
& =\sum_{k^{\prime}=0}^{\infty}\left(\begin{array}{c}
\alpha \\
k^{\prime}
\end{array}\right) \frac{D^{k^{\prime}}\left(f \circ \psi^{-1}\right) \psi(x)}{\Gamma\left(k^{\prime}-\alpha+1\right)}(\psi(x)-\psi(a))^{k^{\prime}-\alpha} .
\end{aligned}
$$


Theorem 25. Let $\beta>0$ and $m=[\beta]+1$ be two reals.

(1) If $f(x)=I_{a+}^{\alpha+\beta, \psi} g(x)$, for some continuous function $g:[a, b] \rightarrow \mathbb{R}$, then

$$
D_{a+}^{\alpha, \psi} D_{a+}^{\beta, \psi} f(x)=D_{a+}^{\alpha+\beta, \psi} f(x) .
$$

(2) If $f(x)=I_{b-}^{\alpha+\beta, \psi} g(x)$, for some continuous function $g:[a, b] \rightarrow \mathbb{R}$, then

$$
D_{b-}^{\alpha, \psi} D_{b-}^{\beta, \psi} f(x)=D_{b-}^{\alpha+\beta, \psi} f(x) .
$$

Proof. Evaluating the left-hand side of equation (7):

$$
\begin{aligned}
D_{a+}^{\alpha, \psi} D_{a+}^{\beta, \psi} f(x) & =\left(\frac{1}{\psi^{\prime}(x)} \frac{d}{d x}\right)^{n} I_{a+}^{n-\alpha, \psi}\left(\frac{1}{\psi^{\prime}(x)} \frac{d}{d x}\right)^{m} I_{a+}^{m-\beta, \psi} I_{a+}^{\alpha+\beta, \psi} g(x) \\
& =\left(\frac{1}{\psi^{\prime}(x)} \frac{d}{d x}\right)^{n} I_{a+}^{n-\alpha, \psi}\left(\frac{1}{\psi^{\prime}(x)} \frac{d}{d x}\right)^{m} I_{a+}^{m, \psi} I_{a+}^{\alpha, \psi} g(x) \\
& =\left(\frac{1}{\psi^{\prime}(x)} \frac{d}{d x}\right)^{n} I_{a+}^{n-\alpha, \psi} I_{a+}^{\alpha, \psi} g(x) \\
& =\left(\frac{1}{\psi^{\prime}(x)} \frac{d}{d x}\right)^{n} I_{a+}^{n, \psi} g(x)=g(x)
\end{aligned}
$$

On the other hand,

$$
D_{a+}^{\alpha+\beta, \psi} f(x)=\left(\frac{1}{\psi^{\prime}(x)} \frac{d}{d x}\right)^{[\alpha+\beta]+1} I_{a+}^{[\alpha+\beta]+1-\alpha-\beta, \psi} I_{a+}^{\alpha+\beta, \psi} g(x)=g(x) .
$$

Therefore,

$$
D_{a+}^{\alpha, \psi} D_{a+}^{\beta, \psi} f(x)=D_{a+}^{\alpha+\beta, \psi} f(x)
$$

Theorem 26. Let $\lambda>-1$ and $\beta \in \mathbb{R}$ with $\beta<\lambda+1$.

(1) If $f(x)=(\psi(x)-\psi(a))^{\lambda} g(\psi(x)-\psi(a))$, for some function $g$ of form $g(x)=\sum_{k=0}^{\infty} a_{n} x^{k}$, then

$$
D_{a+}^{\alpha, \psi} D_{a+}^{\beta, \psi} f(x)=D_{a+}^{\alpha+\beta, \psi} f(x) .
$$

(2) If $f(x)=(\psi(b)-\psi(x))^{\lambda} g(\psi(b)-\psi(x))$, for some function $g$ of form $g(x)=\sum_{k=0}^{\infty} a_{n} x^{k}$, then

$$
D_{b-}^{\alpha, \psi} D_{b-}^{\beta, \psi} f(x)=D_{b-}^{\alpha+\beta, \psi} f(x) .
$$


Proof. Since

$$
f(x)=\sum_{k=0}^{\infty} a_{n}(\psi(x)-\psi(a))^{k+\lambda}
$$

we get that

$$
D_{a+}^{\beta, \psi} f(x)=\sum_{k=0}^{\infty} a_{n} \frac{\Gamma(k+\lambda+1)}{\Gamma(k+\lambda+1-\beta)}(\psi(x)-\psi(a))^{k+\lambda-\beta} .
$$

Since $k+\lambda-\beta>-1$, for all $k \in \mathbb{N} \cup\{0\}$,

$$
\begin{aligned}
D_{a+}^{\alpha, \psi} D_{a+}^{\beta, \psi} f(x) & =\sum_{k=0}^{\infty} a_{n} \frac{\Gamma(k+\lambda+1)}{\Gamma(k+\lambda+1-\beta-\alpha)}(\psi(x)-\psi(a))^{k+\lambda-\beta-\alpha} \\
& =D_{a+\beta, \psi}^{\alpha+\beta} f(x) .
\end{aligned}
$$

Theorem 27. Given a positive integer $m$, we have

$$
\left(\frac{1}{\psi^{\prime}(x)} \frac{d}{d x}\right)^{m} D_{a+}^{\alpha, \psi} f(x)=D_{a+}^{m+\alpha, \psi} f(x)
$$

and

$$
\left(-\frac{1}{\psi^{\prime}(x)} \frac{d}{d x}\right)^{m} D_{b-}^{\alpha, \psi} f(x)=D_{b-}^{m+\alpha, \psi} f(x) .
$$

Proof. It is straightforward.

Theorem 28. Let $f$ be a function of class $C^{m}$, with $m \in \mathbb{N}$. Then,

$$
\begin{aligned}
& D_{a+}^{\alpha, \psi}\left(\frac{1}{\psi^{\prime}(x)} \frac{d}{d x}\right)^{m} f(x) \\
& \quad=D_{a+}^{\alpha+m, \psi} f(x)-\sum_{k=0}^{m-1} \frac{f^{[k]}(a)}{\Gamma(k+1-\alpha-m)}(\psi(x)-\psi(a))^{k-\alpha-m}
\end{aligned}
$$

and

$$
\begin{aligned}
& D_{b-}^{\alpha, \psi}\left(-\frac{1}{\psi^{\prime}(x)} \frac{d}{d x}\right)^{m} f(x) \\
& \quad=D_{b-}^{m+\alpha, \psi} f(x)-\sum_{k=0}^{m-1} \frac{(-1)^{k} f^{[k]}(b)}{\Gamma(k+1-\alpha-m)}(\psi(b)-\psi(x))^{k-\alpha-m} .
\end{aligned}
$$


Proof. Using Theorems 6 and 8, together with Lemma 14, we deduce the following:

$$
\begin{aligned}
D_{a+}^{\alpha, \psi} & \left(\frac{1}{\psi^{\prime}(x)} \frac{d}{d x}\right)^{m} f(x) \\
& =\left(\frac{1}{\psi^{\prime}(x)} \frac{d}{d x}\right)^{n} I_{a+}^{n-\alpha, \psi}\left(\frac{1}{\psi^{\prime}(x)} \frac{d}{d x}\right)^{m} f(x) \\
& =\left(\frac{1}{\psi^{\prime}(x)} \frac{d}{d x}\right)^{n+m} I_{a+}^{m, \psi} I_{a+}^{n-\alpha, \psi}\left(\frac{1}{\psi^{\prime}(x)} \frac{d}{d x}\right)^{m} f(x) \\
& =D_{a+}^{\alpha+m, \psi} I_{a+}^{m, \psi}\left(\frac{1}{\psi^{\prime}(x)} \frac{d}{d x}\right)^{m} f(x) \\
& =D_{a+}^{\alpha+m, \psi}\left(f(x)-\sum_{k=0}^{m-1} \frac{f^{[k]}(a)}{k !}(\psi(x)-\psi(a))^{k}\right) \\
& =D_{a+}^{\alpha+m, \psi} f(x)-\sum_{k=0}^{m-1} \frac{f^{[k]}(a)}{\Gamma(k+1-\alpha-m)}(\psi(x)-\psi(a))^{k-\alpha-m}
\end{aligned}
$$

Therefore, we verify that fractional operator $D_{a+}^{\alpha, \psi}$ commutes with $\left(1 / \psi^{\prime}(x) d / d x\right)^{m}$ only if $f^{[k]}(a)=0$, for every $k=0, \ldots, m-1$.

4. Fractional integrals and fractional derivatives. Similarly to ordinary integrals and ordinary derivatives, fractional integration and fractional differentiation are inverse operations, as we shall see below.

Theorem 29. If $f$ is continuous, then, for every $x$,

$$
D_{a+}^{\alpha, \psi} I_{a+}^{\alpha, \psi} f(x)=D_{b-}^{\alpha, \psi} I_{b-}^{\alpha, \psi} f(x)=f(x) .
$$

Proof. By definition and using Theorem 6, we have

$$
\begin{aligned}
D_{a+}^{\alpha, \psi} I_{a+}^{\alpha, \psi} f(x) & =\left(\frac{1}{\psi^{\prime}(x)} \frac{d}{d x}\right)^{n} I_{a+}^{n-\alpha, \psi} I_{a+}^{\alpha, \psi} f(x) \\
& =\left(\frac{1}{\psi^{\prime}(x)} \frac{d}{d x}\right)^{n} I_{a+}^{n, \psi} f(x)=f(x) .
\end{aligned}
$$


Theorem 30. Let $f \in C^{n}[a, b]$ be a function such that $D_{a+}^{\alpha, \psi} f$ and $D_{b-}^{\alpha, \psi} f$ exist on $[a, b]$. Then,

$$
I_{a+}^{\alpha, \psi} D_{a+}^{\alpha, \psi} f(x)=I_{b-}^{\alpha, \psi} D_{b-}^{\alpha, \psi} f(x)=f(x) .
$$

Proof. Since $f$ is of class $C^{n}$, by (3), we have that

$$
D_{a+}^{\alpha, \psi} f(x)={ }^{C} D_{a+}^{\alpha, \psi} f(x) \text { and } f_{\psi}^{[k]}(a)=0 \text { for all } k=0,1, \ldots, n-1 .
$$

Therefore, by [2, Theorem 4]

$$
I_{a+}^{\alpha, \psi} D_{a+}^{\alpha, \psi} f(x)=I_{a+}^{\alpha, \psi C} D_{a+}^{\alpha, \psi} f(x)=f(x) .
$$

Theorem 31. If $f$ is continuous, then given $m \in \mathbb{N}$ with $m \geq n$, we have

$$
D_{a+}^{\alpha, \psi} f(x)=\left(\frac{1}{\psi^{\prime}(x)} \frac{d}{d x}\right)^{m} I_{a+}^{m-\alpha, \psi} f(x)
$$

and

$$
D_{b-}^{\alpha, \psi} f(x)=\left(-\frac{1}{\psi^{\prime}(x)} \frac{d}{d x}\right)^{m} I_{b-}^{m-\alpha, \psi} f(x) .
$$

Proof. Using the semigroup law for fractional integrals and Theorem 6 , we deduce the following:

$$
\begin{aligned}
\left(\frac{1}{\psi^{\prime}(x)} \frac{d}{d x}\right)^{m} & I_{a+}^{m-\alpha, \psi} f(x) \\
& =\left(\frac{1}{\psi^{\prime}(x)} \frac{d}{d x}\right)^{n}\left(\frac{1}{\psi^{\prime}(x)} \frac{d}{d x}\right)^{m-n} I_{a+}^{m-n, \psi} f(x) I_{a+}^{n-\alpha, \psi} f(x) \\
& =\left(\frac{1}{\psi^{\prime}(x)} \frac{d}{d x}\right)^{n} I_{a+}^{n-\alpha, \psi} f(x)=D_{a+}^{\alpha, \psi} f(x) .
\end{aligned}
$$

Theorem 32. Let $f$ be a continuous function, $\beta>0$ a real with $\alpha+\beta<n$, and $m=[\beta]+1$.

(1) If $D_{a+}^{\beta, \psi} f$ is a continuous function, then

$$
D_{a+}^{\alpha, \psi} D_{a+}^{\beta, \psi} f(x)=D_{a+}^{\alpha+\beta, \psi} f(x)-\sum_{k=1}^{m} \frac{D_{a+}^{\beta-k, \psi} f(a)}{\Gamma(1-\alpha-k)}(\psi(x)-\psi(a))^{-\alpha-k} .
$$


(2) If $D_{b-}^{\beta, \psi} f$ is a continuous function, then

$$
D_{b-}^{\alpha, \psi} D_{b-}^{\beta, \psi} f(x)=D_{b-}^{\alpha+\beta, \psi} f(x)-\sum_{k=1}^{m} \frac{D_{b-}^{\beta-k, \psi} f(b)}{\Gamma(1-\alpha-k)}(\psi(b)-\psi(x))^{-\alpha-k} .
$$

Proof. Using Theorem 30, we get

$$
\begin{aligned}
D_{a+}^{\alpha, \psi} & D_{a+}^{\beta, \psi} f(x) \\
= & \left(\frac{1}{\psi^{\prime}(x)} \frac{d}{d x}\right)^{n} I_{a+}^{n-\alpha, \psi} D_{a+}^{\beta, \psi} f(x) \\
= & \left(\frac{1}{\psi^{\prime}(x)} \frac{d}{d x}\right)^{n} I_{a+}^{n-\alpha-\beta, \psi} I_{a+}^{\beta, \psi} D_{a+}^{\beta, \psi} f(x) \\
= & \left(\frac{1}{\psi^{\prime}(x)} \frac{d}{d x}\right)^{n} I_{a+}^{n-\alpha-\beta, \psi}\left[f(x)-\sum_{k=1}^{m} D_{a+}^{\beta-k, \psi} f(a) \frac{(\psi(x)-\psi(a))^{\beta-k}}{\Gamma(\beta-k+1)}\right] \\
= & \left(\frac{1}{\psi^{\prime}(x)} \frac{d}{d x}\right)^{n} I_{a+}^{n-\alpha-\beta, \psi} f(x) \\
& -\sum_{k=1}^{m} D_{a+}^{\beta-k, \psi} f(a)\left(\frac{1}{\psi^{\prime}(x)} \frac{d}{d x}\right)^{n} \frac{(\psi(x)-\psi(a))^{n-\alpha-k}}{\Gamma(n-\alpha-k+1)} \\
= & D_{a+}^{\alpha+\beta, \psi} f(x)-\sum_{k=1}^{m} \frac{D_{a+}^{\beta-k, \psi} f(a)}{\Gamma(1-\alpha-k)}(\psi(x)-\psi(a))^{-\alpha-k} .
\end{aligned}
$$

We remark that, by Theorem 6 , and using the fact that $n=[\alpha+\beta]+m$, for some $m \in \mathbb{N}$,

$$
\begin{aligned}
& \left(\frac{1}{\psi^{\prime}(x)} \frac{d}{d x}\right)^{n} I_{a+}^{n-\alpha-\beta, \psi} f(x) \\
& \quad=\left(\frac{1}{\psi^{\prime}(x)} \frac{d}{d x}\right)^{[\alpha+\beta]+1}\left(\frac{1}{\psi^{\prime}(x)} \frac{d}{d x}\right)^{m-1} I_{a+}^{m-1, \psi} I_{a+}^{[\alpha+\beta]+1-\alpha-\beta, \psi} f(x) \\
& \quad=\left(\frac{1}{\psi^{\prime}(x)} \frac{d}{d x}\right)^{[\alpha+\beta]+1} I_{a+}^{[\alpha+\beta]+1-\alpha-\beta, \psi} f(x) \\
& =D_{a+}^{\alpha+\beta, \psi} f(x) .
\end{aligned}
$$

5. Expansion formulas for fractional integrals and derivatives. In this section, we present a decomposition formula for the fractional operators, involving integer order derivatives only. This way, 
we can rewrite any given fractional problem depending on a fractional integral or a fractional derivative by replacing the fractional operator with our formula. The result then is an ordinary problem that we can solve by any known standard technique.

Theorem 33. Let $f$ be a function of class $C^{1}$ and $N$ a positive integer. Then, $I_{a+}^{\alpha, \psi} f(x)=A_{N}(\psi(x)-\psi(a))^{\alpha} f(x)-\sum_{k=1}^{N} B_{k}(\psi(x)-\psi(a))^{\alpha-k} V_{k}(t)+\bar{E}_{N}(t)$ and $I_{b-}^{\alpha, \psi} f(x)=A_{N}(\psi(b)-\psi(x))^{\alpha} f(x)-\sum_{k=1}^{N} B_{k}(\psi(b)-\psi(x))^{\alpha-k} W_{k}(t)+\overline{\bar{E}}_{N}(t)$, where

$$
\begin{aligned}
A_{N} & :=\frac{1}{\Gamma(\alpha+1)}\left[1+\sum_{k=1}^{N}(-1)^{k}\left(\begin{array}{l}
\alpha \\
k
\end{array}\right)\right], \\
B_{k} & :=\frac{(-1)^{k}}{\Gamma(\alpha+1)}\left(\begin{array}{l}
\alpha \\
k
\end{array}\right), \quad k=1, \ldots, N, \\
V_{k}(t) & :=\int_{a}^{x} k \psi^{\prime}(t)(\psi(t)-\psi(a))^{k-1} f(t) d t, \quad k=1, \ldots, N, \\
W_{k}(t) & :=\int_{x}^{b} k \psi^{\prime}(t)(\psi(b)-\psi(t))^{k-1} f(t) d t, \quad k=1, \ldots, N,
\end{aligned}
$$

and

$$
\lim _{N \rightarrow \infty} \bar{E}_{N}(t)=\lim _{N \rightarrow \infty} \overline{\bar{E}}_{N}(t)=0 \text { for all } t \in[a, b] .
$$

Proof. Integrating by parts, we obtain the formula

$$
I_{a+}^{\alpha, \psi} f(x)=\frac{f(a)}{\Gamma(\alpha+1)}(\psi(x)-\psi(a))^{\alpha}+\frac{1}{\Gamma(\alpha+1)} \int_{a}^{x}(\psi(x)-\psi(t))^{\alpha} f^{\prime}(t) d t .
$$

Using the binomial theorem, we get that

$$
\begin{aligned}
(\psi(x)-\psi(t))^{\alpha} & =(\psi(x)-\psi(a))^{\alpha}\left(1-\frac{\psi(t)-\psi(a)}{\psi(x)-\psi(a)}\right)^{\alpha} \\
& =\sum_{k=0}^{\infty}(\psi(x)-\psi(a))^{\alpha-k}(-1)^{k}\left(\begin{array}{l}
\alpha \\
k
\end{array}\right)(\psi(t)-\psi(a))^{k} .
\end{aligned}
$$


If we define the error function $\bar{E}_{N}$ by the expression

$$
\begin{aligned}
& \bar{E}_{N}(t) \\
& :=\frac{(\psi(x)-\psi(a))^{\alpha}}{\Gamma(\alpha+1)} \sum_{k=N+1}^{\infty} \frac{(-1)^{k}}{(\psi(x)-\psi(a))^{k}}\left(\begin{array}{l}
\alpha \\
k
\end{array}\right) \int_{a}^{x}(\psi(t)-\psi(a))^{k} f^{\prime}(t) d t,
\end{aligned}
$$

then we obtain

$$
\begin{aligned}
I_{a+}^{\alpha, \psi} f(x)= & \frac{f(a)}{\Gamma(\alpha+1)}(\psi(x)-\psi(a))^{\alpha}+\frac{(\psi(x)-\psi(a))^{\alpha}}{\Gamma(\alpha+1)} \\
& \times \sum_{k=0}^{N} \frac{(-1)^{k}}{(\psi(x)-\psi(a))^{k}}\left(\begin{array}{l}
\alpha \\
k
\end{array}\right) \int_{a}^{x}(\psi(t)-\psi(a))^{k} f^{\prime}(t) d t+\bar{E}_{N}(t) .
\end{aligned}
$$

Decomposing the infinite sum, integrating, and doing another integration by parts allows us to write

$$
\begin{aligned}
I_{a+}^{\alpha, \psi} & f(x) \\
= & \frac{f(a)}{\Gamma(\alpha+1)}(\psi(x)-\psi(a))^{\alpha} \\
& +\frac{(\psi(x)-\psi(a))^{\alpha}}{\Gamma(\alpha+1)} \int_{a}^{x} f^{\prime}(t) d t+\sum_{k=1}^{N} \frac{(-1)^{k}(\psi(x)-\psi(a))^{\alpha-k}}{\Gamma(\alpha+1)}\left(\begin{array}{l}
\alpha \\
k
\end{array}\right) \\
& \times\left[(\psi(x)-\psi(a))^{k} f(x)-\int_{a}^{x} k \psi^{\prime}(t)(\psi(t)-\psi(a))^{k-1} f(t) d t\right]+\bar{E}_{N}(t) \\
= & A_{N}(\psi(x)-\psi(a))^{\alpha} f(x)-\sum_{k=1}^{N} B_{k}(\psi(x)-\psi(a))^{\alpha-k} V_{k}(t)+\bar{E}_{N}(t) .
\end{aligned}
$$

To end the proof, let us see that

$$
\lim _{N \rightarrow \infty} \bar{E}_{N}(t)=0 \text { for all } t \in[a, b] .
$$

For that purpose, let

$$
M:=\max _{t \in[a, b]}\left|f^{\prime}(t)\right| .
$$

Attending that, for all $t \in[a, x]$,

$$
\left|\frac{\psi(t)-\psi(a)}{\psi(x)-\psi(a)}\right| \leq 1
$$


and that (see [31, Equation (1.51)],

as $k \rightarrow \infty$, we deduce that

$$
\left|\left(\begin{array}{l}
\alpha \\
k
\end{array}\right)\right| \leq \frac{c}{k^{1+\alpha}}
$$

$$
\sum_{k=N+1}^{\infty}\left|\left(\begin{array}{l}
\alpha \\
k
\end{array}\right)\right| \leq \int_{N}^{\infty} \frac{c}{k^{\alpha+1}} d k=\frac{c}{\alpha N^{\alpha}},
$$

for $k$ sufficiently large. So, in conclusion, we have the following upperbound:

$$
\left|\bar{E}_{N}(t)\right| \leq M c \frac{(\psi(x)-\psi(a))^{\alpha}(x-a)}{\alpha \Gamma(\alpha+1) N^{\alpha}},
$$

which converges to zero, as $N$ goes to infinity.

We now show the accuracy of the given method. Fix the kernel $\psi(x)=\sqrt[3]{x+1}$, with $x \in[0,10]$, and the functions $f(x)=(\psi(x)-\psi(0))^{3}$ and $g(x)=(\psi(10)-\psi(x))^{3}$. We present the obtained results for the fractional order $\alpha=0.5$ (Figure 3). The continuous curves are the plots of $I_{0+}^{0.5, \psi} f(x)$ and $I_{10-}^{0.5, \psi} g(x)$, given by (1), (2), and the dashed lines are the numerical results from Theorem 33 for different values of $N$. We also display the error of the approximation as the absolute value of the difference between the exact expression and the numerical approximation.

Next, we obtain the expansion formulas for the left and right fractional derivatives.

Theorem 34. Let $f$ be a function of class $C^{n+1}$ and $N \in \mathbb{N}$ with $N \geq n+1$. Then,

$$
\begin{aligned}
D_{a+}^{\alpha, \psi} f(x)=\sum_{k=0}^{n} A_{k, N}(\psi(x) & -\psi(a))^{k-\alpha} f_{\psi}^{[k]}(x) \\
& -\sum_{k=n+1}^{N} B_{k}(\psi(x)-\psi(a))^{n-\alpha-k} V_{k}(t)+\bar{E}_{N}(t)
\end{aligned}
$$

and

$$
\begin{aligned}
D_{b-}^{\alpha, \psi} f(x)=\sum_{k=0}^{n}(-1)^{k} A_{k, N}(\psi(b)-\psi(x))^{k-\alpha} f_{\psi}^{[k]}(x) & \\
& -\sum_{k=n+1}^{N} B_{k}(\psi(b)-\psi(x))^{n-\alpha-k} W_{k}(t)+\overline{\bar{E}}_{N}(t),
\end{aligned}
$$




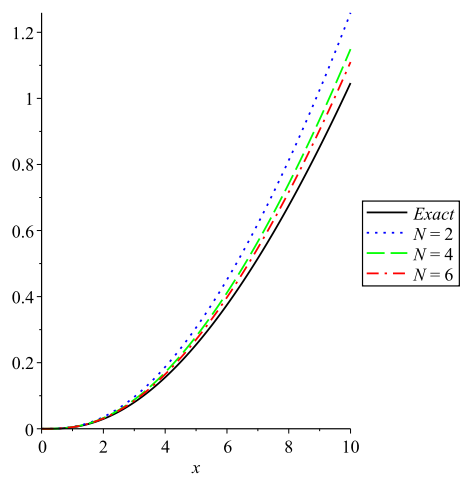

$$
I_{0+}^{0.5, \psi} f(x)
$$

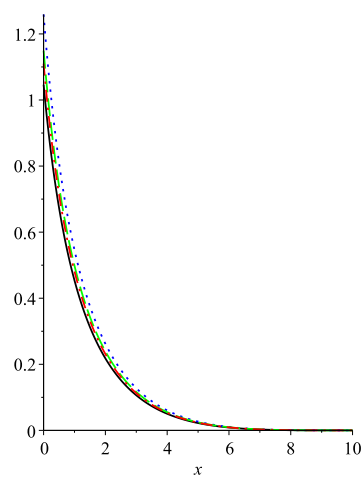

$$
I_{10-}^{0.5, \psi} g(x)
$$

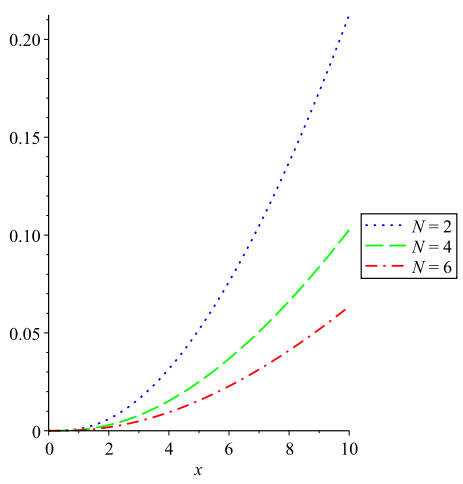

error for $I_{0+}^{0.5, \psi} f(x)$

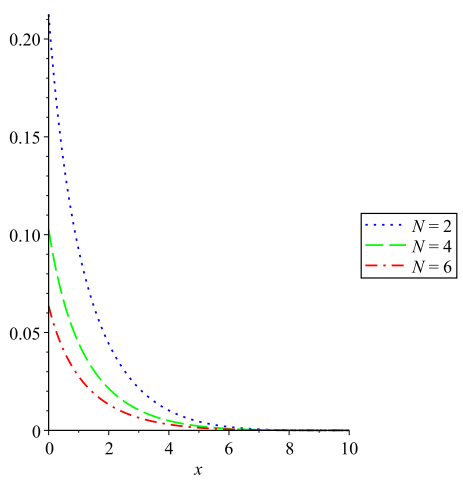

error for $I_{10-}^{0.5, \psi} g(x)$

FiguRE 3. Left and right fractional integrals of order $\alpha=0.5$.

where

$$
\begin{aligned}
A_{k, N} & :=\frac{1}{\Gamma(k-\alpha+1)}\left[1+\sum_{j=n-k+1}^{N}(-1)^{j+k-n}\left(\begin{array}{c}
k-\alpha \\
j+k-n
\end{array}\right)\right], \\
B_{k} & :=\frac{(-1)^{k-n}}{\Gamma(1-\alpha)}\left(\begin{array}{c}
-\alpha \\
k-n
\end{array}\right), \\
V_{k}(t) & :=\int_{a}^{x}(k-n) \psi^{\prime}(t)(\psi(t)-\psi(a))^{k-n-1} f(t) d t \\
W_{k}(t) & :=\int_{x}^{b}(k-n) \psi^{\prime}(t)(\psi(b)-\psi(t))^{k-n-1} f(t) d t
\end{aligned}
$$


and

$$
\lim _{N \rightarrow \infty} \bar{E}_{N}(t)=\lim _{N \rightarrow \infty} \overline{\bar{E}}_{N}(t)=0 \text { for all } t \in[a, b] .
$$

Proof. Starting with (3), and integrating by parts, we arrive at

$$
\begin{aligned}
D_{a+}^{\alpha, \psi} f(x)= & \sum_{k=0}^{n-1} \frac{f_{\psi}^{[k]}(a)}{\Gamma(k+1-\alpha)}(\psi(x)-\psi(a))^{k-\alpha} \\
& \quad+\frac{1}{\Gamma(n-\alpha)} \int_{a}^{x} \psi^{\prime}(t)(\psi(x)-\psi(t))^{n-\alpha-1} f_{\psi}^{[n]}(t) d t \\
= & \sum_{k=0}^{n} \frac{f_{\psi}^{[k]}(a)}{\Gamma(k+1-\alpha)}(\psi(x)-\psi(a))^{k-\alpha} \\
& \quad+\frac{(\psi(x)-\psi(a))^{n-\alpha}}{\Gamma(n+1-\alpha)} \int_{a}^{x}\left(1-\frac{\psi(t)-\psi(a)}{\psi(x)-\psi(a)}\right)^{n-\alpha} \frac{d}{d t} f_{\psi}^{[n]}(t) d t .
\end{aligned}
$$

By the binomial theorem, and splitting the sum and performing an integration by parts, we get

$$
\begin{aligned}
& \begin{array}{l}
D_{a+}^{\alpha, \psi} f(x) \\
=\sum_{k=0}^{n} \frac{f_{\psi}^{[k]}(a)}{\Gamma(k+1-\alpha)}(\psi(x)-\psi(a))^{k-\alpha} \\
\quad+\sum_{k=0}^{N} \frac{(-1)^{k}(\psi(x)-\psi(a))^{n-\alpha-k}}{\Gamma(n+1-\alpha)}\left(\begin{array}{c}
n-\alpha \\
k
\end{array}\right) \\
\quad \times \int_{a}^{x}(\psi(t)-\psi(a))^{k} \frac{d}{d t} f_{\psi}^{[n]}(t) d t+\bar{E}_{N}(t) \\
=\sum_{k=0}^{n} \frac{f_{\psi}^{[k]}(a)}{\Gamma(k+1-\alpha)}(\psi(x)-\psi(a))^{k-\alpha}+\frac{(\psi(x)-\psi(a))^{n-\alpha}}{\Gamma(n+1-\alpha)} \int_{a}^{x} \frac{d}{d t} f_{\psi}^{[n]}(t) d t \\
+\sum_{k=1}^{N} \frac{(-1)^{k}(\psi(x)-\psi(a))^{n-\alpha-k}}{\Gamma(n+1-\alpha)}\left(\begin{array}{c}
n-\alpha \\
k
\end{array}\right) \\
\quad \times\left[(\psi(x)-\psi(a))^{k} f_{\psi}^{[n]}(x)\right. \\
\left.\quad-\int_{a}^{x} k \psi^{\prime}(t)(\psi(t)-\psi(a))^{k-1} f_{\psi}^{[n]}(t)\right] d t+\bar{E}_{N}(t)
\end{array}
\end{aligned}
$$




$$
\begin{gathered}
=\sum_{k=0}^{n-1} \frac{f_{\psi}^{[k]}(a)}{\Gamma(k+1-\alpha)}(\psi(x)-\psi(a))^{k-\alpha}+A_{n, N}(\psi(x)-\psi(a))^{n-\alpha} f_{\psi}^{[n]}(x) \\
-\sum_{k=1}^{N} \frac{(-1)^{k}(\psi(x)-\psi(a))^{n-\alpha-k}}{\Gamma(n-\alpha)}\left(\begin{array}{c}
n-\alpha-1 \\
k-1
\end{array}\right) \\
\quad \times \int_{a}^{x}(\psi(t)-\psi(a))^{k-1} \frac{d}{d t} f_{\psi}^{[n-1]}(t) d t+\bar{E}_{N}(t),
\end{gathered}
$$

where

$$
\begin{aligned}
& \bar{E}_{N}(t):=\frac{(\psi(x)-\psi(a))^{n-\alpha}}{\Gamma(n+1-\alpha)} \\
& \quad \times \sum_{k=N+1}^{\infty}(-1)^{k}\left(\begin{array}{c}
n-\alpha \\
k
\end{array}\right) \int_{a}^{x}\left(\frac{\psi(t)-\psi(a)}{\psi(x)-\psi(a)}\right)^{k} \frac{d}{d t} f_{\psi}^{[n]}(t) d t .
\end{aligned}
$$

Observe that

$$
\begin{aligned}
\frac{k}{\Gamma(n+1-\alpha)}\left(\begin{array}{c}
n-\alpha \\
k
\end{array}\right) & =\frac{k}{\Gamma(n+1-\alpha)} \frac{\Gamma(n+1-\alpha)}{\Gamma(n-\alpha-k+1) k !} \\
& =\frac{1}{\Gamma(n-\alpha)}\left(\begin{array}{c}
n-\alpha-1 \\
k-1
\end{array}\right) .
\end{aligned}
$$

Splitting the sum again into the first term $k=1$ and the remaining terms $k=2, \ldots, N$, and then using integration by parts, we get

$$
\begin{aligned}
D_{a+}^{\alpha, \psi} f(x)=\sum_{k=0}^{n-1} & \frac{f_{\psi}^{[k]}(a)}{\Gamma(k+1-\alpha)}(\psi(x)-\psi(a))^{k-\alpha} \\
& +A_{n, N}(\psi(x)-\psi(a))^{n-\alpha} f_{\psi}^{[n]}(x) \\
& +\frac{(\psi(x)-\psi(a))^{n-\alpha-1}}{\Gamma(n-\alpha)} \int_{a}^{x} \frac{d}{d t} f_{\psi}^{[n-1]}(t) d t \\
& -\sum_{k=2}^{N} \frac{(-1)^{k}(\psi(x)-\psi(a))^{n-\alpha-k}}{\Gamma(n-\alpha)}\left(\begin{array}{c}
n-\alpha-1 \\
k-1
\end{array}\right) \\
& \times\left[(\psi(x)-\psi(a))^{k-1} f_{\psi}^{[n-1]}(x)\right. \\
& \left.\quad-\int_{a}^{x}(k-1) \psi^{\prime}(t)(\psi(t)-\psi(a))^{k-2} f_{\psi}^{[n-1]}(t)\right] d t+\bar{E}_{N}(t)
\end{aligned}
$$




$$
\begin{aligned}
=\sum_{k=0}^{n-2} \frac{f_{\psi}^{[k]}(a)}{\Gamma(k+1-\alpha)}(\psi(x)-\psi(a))^{k-\alpha} & \\
& +\sum_{k=n-1}^{n} A_{k, N}(\psi(x)-\psi(a))^{k-\alpha} f_{\psi}^{[k]}(x) \\
& -\sum_{k=2}^{N} \frac{(-1)^{k-1}(\psi(x)-\psi(a))^{n-\alpha-k}}{\Gamma(n-\alpha-1)}\left(\begin{array}{c}
n-\alpha-2 \\
k-2
\end{array}\right) \\
& \times \int_{a}^{x}(\psi(t)-\psi(a))^{k-2} \frac{d}{d t} f_{\psi}^{[n-2]}(t) d t+\bar{E}_{N}(t) .
\end{aligned}
$$

Repeating this procedure, we arrive at the desired formula. For the error, let $M:=\max _{t \in[a, b]}\left|\frac{d}{d t} f_{\psi}^{[n]}(t)\right|$. Then,

$$
\left|\bar{E}_{N}(t)\right| \leq M c \frac{(\psi(x)-\psi(a))^{n-\alpha}(x-a)}{(n-\alpha) \Gamma(n-\alpha+1) N^{n-\alpha}},
$$

and so $\lim _{N \rightarrow \infty} \bar{E}_{N}(t)=0$.

To test the result, we again consider $\psi(x)=\sqrt[3]{x+1}$, with $x \in[0,10]$, and the function $f(x)=(\psi(x)-\psi(0))^{3}$. In Figure 4, we exhibit the graphs of the left fractional derivatives for order $\alpha=0.5$ with three different values of $N$. We do the same in Figure 5 for $\alpha=1.5$.
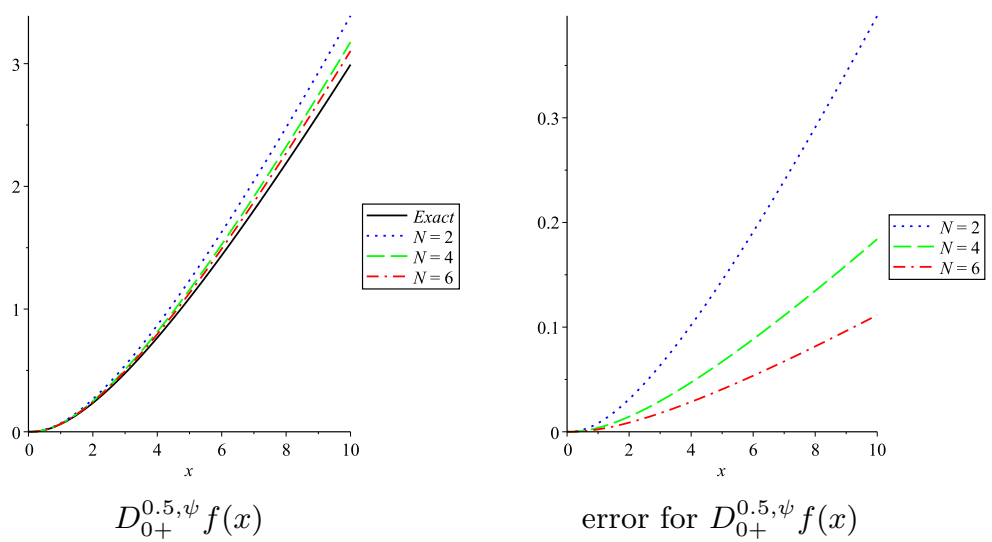

FiguRE 4. Left fractional derivatives of order $\alpha=0.5$. 

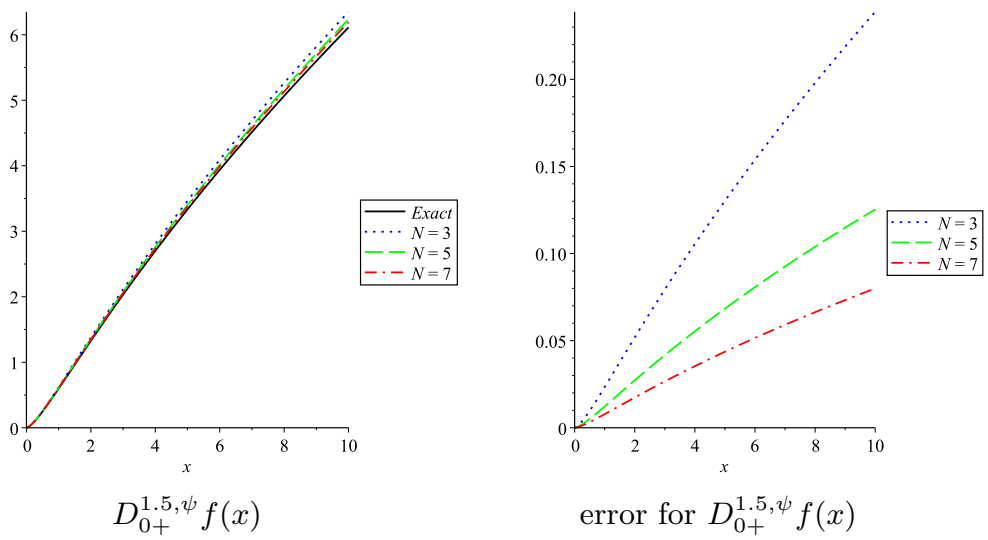

FiguRE 5. Left fractional derivatives of order $\alpha=1.5$.

\section{REFERENCES}

1. M.S. Abdelouahab and N.E. Hamri, The Grünwald-Letnikov fractional-order derivative with fixed memory length, Mediterr. J. Math. 13 (2016), 557-572.

2. R. Almeida, A Caputo fractional derivative of a function with respect to another function, Commun. Nonlinear Sci. Numer. Simul. 44 (2017), 460-481.

3. M.A. Balci, Fractional virus epidemic model on financial networks, Open Math. 14 (2016), no. 1, 1074-1086.

4. P.L. Butzer, A.A. Kilbas and J.J. Trujillo, Fractional calculus in the Mellin setting and Hadamard-type fractional integrals, J. Math. Anal. Appl. 269 (2002), no. $1,1-27$.

5. P.L. Butzer, A.A. Kilbas and J.J. Trujillo, Compositions of Hadamard-type fractional integration operators and the semigroup property, J. Math. Anal. Appl. 269 (2002), no. 2, 387-400.

6. L.M.B.C. Campos, On generalizations of the series of Taylor, Lagrange, Laurent and Teixeira, Internat. J. Math. Math. Sci. 13 (1990), no. 4, 687-708.

7. J.M. Carcione, Theory and modeling of constant- $Q P$ - and $S$-waves using fractional time derivatives, Geophys. 74 (2009), no. 1, T1-T11.

8. W. Chen and G. Pang, A new definition of fractional Laplacian with application to modeling three-dimensional nonlocal heat conduction, J. Comput. Phys. 309 (2016), 350-367.

9. Y. Cho, I. Kim and D. Sheen, A fractional-order model for MINMOD millennium, Math. Biosci. 262 (2015), 36-45.

10. A. El-Ajou, O.A. Arqub, Z.A. Zhour and S. Momani, New results on fractional power series: theories and applications, Entropy 15 (2013), 5305-5323.

11. S. Gaboury and R. Tremblay, Summation formulas obtained by means of 
the generalized chain rule for fractional derivatives, J. Complex Anal. (2014), art. id. 820951, 7 pp.

12. N.M. Grahovac and M.M. Žigić, Modelling of the hamstring muscle group by use of fractional derivatives, Comput. Math. Appl. 59 (2010), no. 5, 1695-1700.

13. F. Jarad, T. Abdeljawad and D. Baleanu, Caputo-type modification of the Hadamard fractional derivatives, Adv. Difference Equ. (2012), art. id. 142, 8 pp.

14. S.L. Kalla and V.S. Kiryakova, An $H$-function generalized fractional calculus based upon compositions of Erdélyi-Kober operators in $L_{p}$, Math. Japonica 35 (1990), 1-21.

15. L. Khitri-Kazi-Tani and H. Dib, On the approximation of Riemann-Liouville integral by fractional nabla h-sum and applications, Mediterr. J. Math. 14 (2017), no. 2 , art. id. 86,21 pp.

16. A.A. Kilbas, H.M. Srivastava and J.J. Trujillo, Theory and applications of fractional differential equations, North-Holland Mathematics Studies 204, Elsevier Science B.V., Amsterdam (2006).

17. J.-L. Lavoie, T.J. Osler and R. Tremblay, Fundamental properties of fractional derivatives via Pochhammer integrals, pp. 323-356 in Fractional calculus and its applications (West Haven, Connecticut, 1974), Lecture Notes in Mathematics 457, Springer (1975).

18. C. Li, D. Qian and Y.Q. Chen, On Riemann-Liouville and Caputo derivatives, Discrete Dyn. Nat. Soc. (2011), art. id. 562494, 15 pp.

19. R.L. Magin and M. Ovadia, Modeling the cardiac tissue electrode interface using fractional calculus, J. Vib. Control 14 (2008), no. 9-10, 1431-1442.

20. S. Min, Some algebra of Leibniz rule for fractional calculus, Int. J. Innov. Sci. Math. 4 (2016), no. 6, 204-208.

21. M.A. Moreles and R. Lainez, Mathematical modelling of fractional order circuit elements and bioimpedance applications, Commun. Nonlinear Sci. Numer. Simul. 46 (2017), 81-88.

22. T.J. Osler, Leibniz rule for fractional derivatives and an application to infinite series, SIAM J. Appl. Math. 18 (1970), 658-674.

23. T.J. Osler, The fractional derivative of a composite function, SIAM J. Math. Anal. 1 (1970), 288-293.

24. T.J. Osler, Leibniz the chain rule and Taylor's theorem for fractional derivatives. Doctoral thesis. New York University (1970).

25. T.J. Osler, Taylor's series generalized for fractional derivatives and applications, SIAM J. Math. Anal. 2 (1971), 37-48.

26. T.J. Osler, A further extension of the Leibniz rule to fractional derivatives and its relation to Parseval's formula, SIAM J. Math. Anal. 3 (1972), 1-16.

27. T.J. Osler, A correction to Leibniz rule for fractional derivatives, SIAM J. Math. Anal. 4 (1973), 456-459.

28. E. Reyes-Melo, J. Martinez-Vega, C. Guerrero-Salazar and U. Ortiz-Mendez, Application of fractional calculus to the modeling of dielectric relaxation phenomena in polymeric materials, J. Appl. Polymer Sci. 98 (2005), no. 2, 923-935. 
29. H.E. Roman and M. Porto, Fractional derivatives of random walks: time series with long-time memory, Phys. Rev. E 78 (2008), art. id. 031127.

30. Y.A. Rossikhin and M.V. Shitikova, Analysis of two colliding fractionally damped spherical shells in modelling blunt human head impacts, Cent. Eur. J. Phys. 11 (2013), no. 6, 760-778.

31. S.G. Samko, A.A. Kilbas and O.I. Marichev, Fractional integrals and derivatives. Translated from the 1987 Russian original. Gordon and Breach, Yverdon (1993).

32. I.N. Sneddon, The use in mathematical analysis of Erdélyi-Kober operators and some of their applications, pp. 37-79 in Fractional calculus and its applications (West Haven, Connecticut, 1974), Lecture Notes in Math. 457, Springer (1975).

33. N.M. Temme, Computational aspects of incomplete gamma functions with large complex parameters, pp. 551-562 in Approximation and computation (West Lafayette, Indiana, 1993), R.V.M. Zahar ed., Birkhäuser, Boston (1994).

34. R. Tremblay, Une contribution à la théorie de la dérivée fractionnaire, $\mathrm{Ph} . \mathrm{D}$. thesis, Laval University, Canada (1974).

35. J.J. Trujillo and M. Rivero and B. Bonilla, On a Riemann-Liouville generalized Taylor's formula, J. Math. Anal. Appl. 231 (1999), no. 1, 255-265.

Center for Research and Development in Mathematics and Applications (CIDma), Department of Mathematics, University of Aveiro, Aveiro, PorTUGAL

Email address: ricardo.almeida@ua.pt 